\title{
Generalized Forbidden Subposet Problems
}

\author{
Dániel Gerbner ${ }^{1} \cdot$ Balázs Keszegh ${ }^{1} \cdot$ Balázs Patkós $^{1}$
}

Received: 5 June 2018 / Accepted: 11 October 2019 / Published online: 10 February 2020

(C) The Author(s) 2020

\begin{abstract}
A subfamily $\left\{F_{1}, F_{2}, \ldots, F_{|P|}\right\} \subseteq \mathcal{F}$ of sets is a copy of a poset $P$ in $\mathcal{F}$ if there exists a bijection $\phi: P \rightarrow\left\{F_{1}, F_{2}, \ldots, F_{|P|}\right\}$ such that whenever $x \leq{ }_{P} x^{\prime}$ holds, then so does $\phi(x) \subseteq \phi\left(x^{\prime}\right)$. For a family $\mathcal{F}$ of sets, let $c(P, \mathcal{F})$ denote the number of copies of $P$ in $\mathcal{F}$, and we say that $\mathcal{F}$ is $P$-free if $c(P, \mathcal{F})=0$ holds. For any two posets $P, Q$ let us denote by $\operatorname{La}(n, P, Q)$ the maximum number of copies of $Q$ over all $P$-free families $\mathcal{F} \subseteq 2^{[n]}$, i.e. $\max \left\{c(Q, \mathcal{F}): \mathcal{F} \subseteq 2^{[n]}, c(P, \mathcal{F})=0\right\}$. This generalizes the well-studied parameter $\operatorname{La}(n, P)=\operatorname{La}\left(n, P, P_{1}\right)$ where $P_{1}$ is the one element poset, i.e. $\operatorname{La}(n, P)$ is the largest possible size of a $P$-free family. The quantity $\operatorname{La}(n, P)$ has been determined (precisely or asymptotically) for many posets $P$, and in all known cases an asymptotically best construction can be obtained by taking as many middle levels as possible without creating a copy of $P$. In this paper we consider the first instances of the problem of determining $\operatorname{La}(n, P, Q)$. We find its value when $P$ and $Q$ are small posets, like chains, forks, the $N$ poset and diamonds. Already these special cases show that the extremal families are completely different from those in the original $P$-free cases: sometimes not middle or consecutive levels maximize $\operatorname{La}(n, P, Q)$ and sometimes the extremal family is not the union of levels. Finally, we determine (up to a polynomial factor) the maximum number of copies of complete multi-level posets in $k$-Sperner families. The main tools for this are the profile polytope method and two extremal set system problems that are of independent interest: we maximize the number of $r$-tuples $A_{1}, A_{2}, \ldots, A_{r} \in \mathcal{A}$ over all antichains $\mathcal{A} \subseteq 2^{[n]}$ such that (i) $\cap_{i=1}^{r} A_{i}=\emptyset$, (ii) $\cap_{i=1}^{r} A_{i}=\emptyset$ and $\cup_{i=1}^{r} A_{i}=[n]$.
\end{abstract}

Keywords Extremal set systems · Forbidden subposet problems

Balázs Patkós

patkos@renyi.hu

Dániel Gerbner

gerbner@renyi.hu

Balázs Keszegh

keszegh@ @enyi.hu

1 Alfréd Rényi Institute of Mathematics, Hungarian Academy of Sciences, P.O.B. 127, Budapest, H-1364, Hungary 


\section{Introduction}

The very first theorem of extremal finite set theory is due to Sperner [25] and states that if $\mathcal{F}$ is a family of subsets of $[n]=\{1,2, \ldots, n\}$ such that no two sets in $\mathcal{F}$ are comparable, then $|\mathcal{F}| \leq\left(\begin{array}{c}n \\ \lceil n / 2\rceil\end{array}\right)$ holds, and equality is achieved if and only if $\mathcal{F}$ consists of all the $\lceil n / 2\rceil$ element or all the $\lfloor n / 2\rfloor$-element subsets of $[n]$. For $0 \leq k \leq n$ the family consisting of all the $k$-element subsets of $[n]$ are called (full) levels and we introduce the notation $\left(\begin{array}{c}{[n]} \\ k\end{array}\right)=\{F \subseteq[n]:|F|=k\}$ for them. Sperner's theorem was generalized by Erdôs [6] to the case when $\mathcal{F}$ is not allowed to contain $k+1$ mutually comparable sets, i.e. a $(k+1)$-chain. He showed that among such families the ones consisting of $k$ middle levels are the largest. In the early eighties, Katona and Tarján [21] introduced a generalization of the problem and started to consider determining the size of the largest family of subsets of $[n]$ that does not contain a configuration defined by inclusions. Such problems are known as forbidden subposet problems and are widely studied (see the recent survey [15] or Chapter 7 of [14]).

In this paper, we propose even further generalizations: we are interested in the maximum number of copies of a given configuration $Q$ in families that do not contain a forbidden subposet $P$. Before giving the precise definitions, let us mention that similar problems were studied by Alon and Shikhelman [1] in the context of graphs. They considered the problem of finding the most number of copies of a graph $T$ that an $H$-free graph can contain.

Definition Let $P$ be an arbitrary poset and $\mathcal{F}$ a family of sets. We say that $\mathcal{G} \subseteq \mathcal{F}$ is a copy of $P$ in $\mathcal{F}$ if there exists a bijection $\phi: P \rightarrow \mathcal{G}$ such that whenever $x \leq{ }_{P} x^{\prime}$ holds, then so does $\phi(x) \subseteq \phi\left(x^{\prime}\right)$. In this case we also say that $P$ is a subposet of $\mathcal{F}$. Let $c(P, \mathcal{F})$ denote the number of copies of $P$ in $\mathcal{F}$ and for any pair of posets $P, Q$, let us define

$$
L a(n, P, Q)=\max \left\{c(Q, \mathcal{F}): \mathcal{F} \subseteq 2^{[n]}, c(P, \mathcal{F})=0\right\},
$$

and for families of posets $\mathcal{P}, \mathcal{Q}$ let us define

$$
L a(n, \mathcal{P}, \mathcal{Q})=\max \left\{\sum_{Q \in \mathcal{Q}} c(Q, \mathcal{F}): \mathcal{F} \subseteq 2^{[n]}, \forall P \in \mathcal{P} \quad c(P, \mathcal{F})=0\right\} .
$$

We denote by $P_{k}$ the chain of length $k$, i.e. the completely ordered poset on $k$ elements. In particular, $P_{1}$ is the poset with one element. Let us state Erdős's above mentioned result with our notation.

Theorem 1.1 (Sperner [25] for $k=1$, Erdős [6] for general $k$ ) For every positive integer $k$ the following holds:

$$
L a\left(n, P_{k+1}, P_{1}\right)=\sum_{i=1}^{k}\left(\begin{array}{c}
n \\
\left\lfloor\frac{n-k}{2}\right\rfloor+i
\end{array}\right) .
$$

The main conjecture of the area of forbidden subposet problems was first published by Griggs and Lu in [16] and by Bukh [3], although it was already folklore by that time in the extremal finite set theory community.

Conjecture 1.2 For a poset $P$ let us denote by $e(P)$ the largest integer $m$ such that for any $n$, any family $\mathcal{F} \subseteq 2^{[n]}$ consisting of $m$ consecutive levels is $P$-free. Then

$$
\lim _{n \rightarrow \infty} \frac{\operatorname{La}\left(n, P, P_{1}\right)}{\left(\begin{array}{c}
n \\
\lfloor n / 2\rfloor
\end{array}\right)}=e(P)
$$


holds.

In other words, Conjecture 1.2 states that to obtain an asymptotically largest $P$-free family $\mathcal{F} \subseteq 2^{[n]}$, one has to consider as many middle levels of $2^{[n]}$ as possible without creating a copy of $P$.

There are not many results in the literature where other posets are counted. Katona [19] determined the maximum number of 2-chains (copies of $\left.P_{2}\right)$ in a 2-Sperner $\left(P_{3}\right.$-free) family $\mathcal{F} \subseteq 2^{[n]}$. This was reproved in [24] and generalized by Gerbner and Patkós in [13].

Theorem 1.3 ([13]) For any $l>k$ the quantity $L a\left(n, P_{l}, P_{k}\right)$ is attained for some family $\mathcal{F}$ that is the union of $l-1$ levels. Moreover, $L a\left(n, P_{k+1}, P_{k}\right)=\left(\begin{array}{l}n \\ i_{k}\end{array}\right) \cdot\left(\begin{array}{c}i_{k} \\ i_{k-1}\end{array}\right) \cdots \cdots\left(\begin{array}{l}i_{2} \\ i_{1}\end{array}\right)$, where $i_{1}<i_{2}<\cdots<i_{k}<n$ are chosen arbitrarily such that the values $i_{1}, i_{2}-i_{1}, i_{3}-$ $i_{2}, \ldots, i_{k}-i_{k-1}, n-i_{k}$ differ by at most one.

Theorem 1.3 shows that to make Conjecture 1.2 valid in this more general context one has to remove at least the word consecutive. But there are cases when all families consisting of full levels are very far from being optimal. Let the generalized diamond poset $D_{k}$ be the poset on $k+2$ elements $a, b_{1}, b_{2}, \ldots, b_{k}, c$ with $a<b_{1}, b_{2}, \ldots, b_{k}<c$. Let us consider $L a\left(n, D_{2}, P_{3}\right)$, which is the maximum number of 3-chains in diamond-free families. Every family that contains at least three full levels of $2^{[n]}$ contains a copy of $D_{2}$, while a family that is the union of at most two levels, does not contain any copy of $P_{3}$. Therefore, if $\mathcal{F}$ is $D_{2}$-free and is the union of full levels, then $c\left(P_{3}, \mathcal{F}\right)=0$, while there are $D_{2}$-free families with lots of copies of $P_{3}$. Note that $D_{2}$ is the smallest poset for which Conjecture 1.2 has not been proved.

Theorem 1.4 For the generalized diamond posets and integers $k>l$ the following holds:

$$
\left(\begin{array}{c}
k-1 \\
l
\end{array}\right) \operatorname{La}\left(n-k+1, P_{3}, P_{2}\right) \leq \operatorname{La}\left(n, D_{k}, D_{l}\right) \leq\left(\left(\begin{array}{c}
k+1 \\
2
\end{array}\right)-k\right)\left(\begin{array}{c}
k-1 \\
l
\end{array}\right) \operatorname{La}\left(n, P_{3}, P_{2}\right) .
$$

Note that Theorem 1.4 implies $\operatorname{La}\left(n, D_{k}, D_{l}\right)=\theta_{k, l}\left(\operatorname{La}\left(n, P_{3}, P_{2}\right)\right)$ for any fixed $k$ and $l$. So it is a natural question whether the $\operatorname{limit} d_{k, l}=\lim _{n \rightarrow \infty} \frac{\operatorname{La}\left(n, D_{k}, D_{l}\right)}{\operatorname{La}\left(n, P_{3}, P_{2}\right)}$ exists and if so, what its value is. In the simplest case $k=2, l=1$ the above inequalities and Theorem 1.3 imply $1 / 3 \leq d_{2,1} \leq 1$.

Let us examine what version of Conjecture 1.2 may hold in our setting. Let $l(P)$ be the height of a poset $P$, i.e. the length of the longest chain in $P$. Clearly, if $\mathcal{F}$ is the union of any $l(P)-1$ full levels, it must be $P$-free. On the other hand if $\mathcal{F}_{n}=\cup_{j=1}^{l(Q)}\left(\begin{array}{c}{[n]} \\ i_{j}\end{array}\right)$ is the union of $l(Q)$ full levels with $i_{j+1}-i_{j} \geq c n$ for some constant $c$ for all $j=1,2, \ldots l(Q)-1$, then $\mathcal{F}_{n}$ contains many copies of $Q$. Therefore we propose the following.

Conjecture 1.5 For any pair $P, Q$ of posets with $l(P)>l(Q)$ there exist an integer $m$ and a sequence of $P$-free families $\mathcal{F}_{n} \subseteq 2^{[n]}$ that are of the form $\mathcal{F}_{n}=\cup_{j=1}^{m}\left(\begin{array}{c}{[n]} \\ i_{j}\end{array}\right)$ such that

$$
L a(n, P, Q)=(1+o(1)) c\left(Q, \mathcal{F}_{n}\right)
$$

holds.

As we will see, this conjecture often holds even if $l(P) \leq l(Q)$. We say that for a pair $P, Q$ of posets Conjecture 1.5 strongly holds if for large enough $n$ we have $\operatorname{La}(n, P, Q)=$ $c\left(Q, \mathcal{F}_{n}\right)$ and almost holds if $\operatorname{La}(n, P, Q)=O\left(n^{k} c\left(Q, \mathcal{F}_{n}\right)\right)$ for some $k$ that depends 
only on $P$ and $Q$. In both cases we also assume the family $\mathcal{F}_{n}$ is $P$-free and is the union of full levels, but we do not assume anything about $l(P)$ and $l(Q)$. Note that the value of $L a(n, P, Q)$ typically grows exponentially in $n$.

In this paper, we address the first instances of the general problem. Let us note that since the first version of this paper, Gerbner, Methuku, Nagy, Patkós and Vizer [12] studied the special case when $Q$ is a chain. We will consider the following posets (see Fig. 1): let $\bigvee_{r}$ denote the poset on $r+1$ elements $0, a_{1}, a_{2}, \ldots, a_{r}$ with $0 \leq a_{i}$ for all $i=1,2, \ldots, r$ and we write $\bigvee$ for $\bigvee_{2}$. Similarly, let $\bigwedge_{r}$ denote the poset on $r+1$ elements $a_{1}, a_{2}, \ldots, a_{r}, 1$ with $a_{i} \leq 1$ for all $i=1,2, \ldots, r$ and we write $\bigwedge$ for $\wedge_{2}$. The poset $N$ contains four elements $a, b, c, d$ with $a \leq c$ and $b \leq c, d$. The butterfly poset $B$ consists of four elements $a, b, c, d$ with $a, b \leq c, d$.

A family that does not contain $P_{2}$ is called an antichain. A family $\mathcal{F}$ that does not contain $P_{k}$ can be easily partitioned into $k-1$ antichains $\mathcal{F}_{1}, \ldots, \mathcal{F}_{k-1}$ the following way: let $\mathcal{F}_{i}$ be the set of minimal elements of $\mathcal{F} \backslash \cup_{j=1}^{i-1} \mathcal{F}_{j}$. We call this the canonical partition of $\mathcal{F}$.

The proof of the next theorem (and of the other theorems) will follow in later sections.

Theorem 1.6 (a) $\operatorname{La}\left(n, \bigvee, P_{2}\right)=\operatorname{La}\left(n, \bigwedge, P_{2}\right)=\left(\begin{array}{c}n \\ \lfloor n / 2\rfloor\end{array}\right)$.

(b) $\quad L a\left(n,\{\bigvee, \bigwedge\}, P_{2}\right)=\left(\begin{array}{c}n-1 \\ \lfloor(n-1) / 2\rfloor\end{array}\right)$.

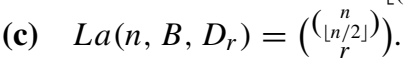

(d) $\operatorname{La}\left(n, \bigvee, \bigwedge_{r}\right)=\operatorname{La}\left(n, \bigwedge, \bigvee_{r}\right)=\left(\begin{array}{c}\left(\begin{array}{c}n \\ \lfloor n / 2\rfloor\end{array}\right) \\ r\end{array}\right)$.

Parts (a) (c), and (d) of Theorem 1.6 show that Conjecture 1.5 strongly holds for those pairs of posets.

The proof of our next theorem uses the notion of profile vectors (ordinary and $l$-chain profile vectors) that we will describe later. Here and throughout the paper $h(x)$ denotes the binary entropy function, i.e. $h(x)=-x \log _{2} x-(1-x) \log _{2}(1-x)$.

Theorem 1.7 (a) $\left.\operatorname{La}\left(n, P_{3}, \bigwedge_{r}\right)=\operatorname{La}\left(n, P_{3}, \bigvee_{r}\right)=\left(\begin{array}{l}n \\ i_{r}\end{array}\right)\left(\underset{r}{\left({ }_{i r}^{i} / 2\right\rfloor}\right)\right)$ for some $i_{r}$ with $i_{r}=$ $(1+o(1)) \frac{2^{r}}{2^{r}+1} n$.

(b) $\quad L a\left(n, P_{4}, D_{r}\right)=\left(\begin{array}{c}n \\ j_{r}\end{array}\right)\left(\begin{array}{l}j_{r} \\ i_{r}\end{array}\right)\left(\begin{array}{c}\left(\begin{array}{c}j_{r}-i_{r} \\ \left.\left.L j_{r}\right) / 2\right\rfloor\end{array}\right) \\ r\end{array}\right)$ for some $i_{r}=(1+o(1)) \frac{n}{2^{r / 2}+2}$ and either $j_{r}=$ $n-i_{r}$ or $j_{r}=n-i_{r}-1$.

(c) $2^{(c+o(1)) n} \leq \operatorname{La}\left(n, P_{3}, N\right) \leq o\left(2^{3 n}\right)$,

where $c=h\left(c_{0}\right)+3 c_{0} h\left(c_{0} /\left(1-c_{0}\right)\right) \approx 2.9502 \ldots$ with $c_{0}$ being the real root of the equation $0=7 x^{3}-10 x^{2}+5 x-1$.

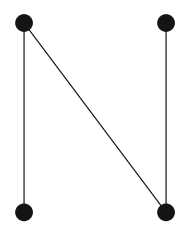

$N$

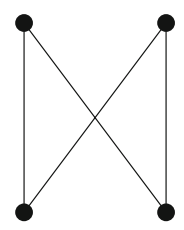

$B$

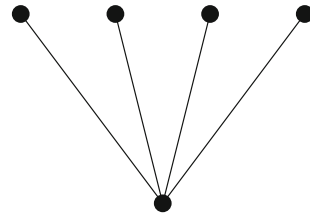

$\bigvee_{r}(r=4)$

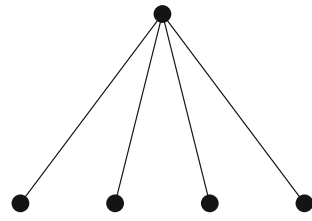

$\bigwedge_{r}(r=4)$

Fig. 1 The Hasse diagrams of the posets $N, B, \bigvee_{r}$ and $\bigwedge_{r}$ 
In the above theorem we dealt with $L a\left(n, P_{l(Q)+1}, Q\right)$ for different posets $Q$. We knew the place of every element of every copy of $Q$ in the canonical partition. In the following we deal with these kind of problems. We introduce the following binary operations of posets: for any pair $Q_{1}, Q_{2}$ of posets we define $Q_{1} \otimes_{r} Q_{2}$ by adding an antichain of size $r$ between $Q_{1}$ and $Q_{2}$. More precisely, let us assume $Q_{1}$ consists of $q_{1}^{1}, \ldots, q_{a}^{1}$ and $Q_{2}$ consists of $q_{1}^{2}, \ldots, q_{b}^{2}$. Then $R=Q_{1} \otimes_{r} Q_{2}$ consists of $q_{1}^{1}, \ldots, q_{a}^{1}, m_{1}, m_{2}, \ldots, m_{r}, q_{1}^{2}, \ldots q_{b}^{2}$. We have $q_{i}^{1}<R q_{j}^{1}$ if and only if $q_{i}^{1}<Q_{1} q_{j}^{1}$ and similarly $q_{i}^{2}<R q_{j}^{2}$ if and only if $q_{i}^{2}<Q_{2} q_{j}^{2}$. Also we have $q_{i}^{1}<_{R} m_{k}<_{R} q_{j}^{2}$ for every $i, k$, and $j$. Finally, the $m_{k}$ 's form an antichain. Note that $l\left(Q_{1} \otimes_{r} Q_{2}\right)=l\left(Q_{1}\right)+l\left(Q_{2}\right)+1$. Let $Q \oplus r$ denote the poset obtained from $Q$ by adding $r$ elements that form an antichain and that are all larger than all elements of $Q$. Similar operations of posets were considered first in the area of forbidden subposet problems by Burcsi and Nagy [4].

We will obtain bounds on $L a\left(n, P_{l\left(Q_{1} \otimes_{r} Q_{2}\right)+1}, Q_{1} \otimes_{r} Q_{2}\right)$ involving bounds on $\operatorname{La}\left(n, P_{l\left(Q_{1}\right)+1}, Q_{1}\right)$ and $\operatorname{La}\left(n, P_{l\left(Q_{2}\right)+1}, Q_{2}\right)$. For this, first we need to introduce the notions of profile vectors and profile polytopes. For a family $\mathcal{F} \subseteq 2^{[n]}$ of sets, let $\alpha(\mathcal{F})=\left(\alpha_{0}, \alpha_{1}, \ldots, \alpha_{n}\right)$ denote the profile vector of $\mathcal{F}$, where $\alpha_{i}=|\{F \in \mathcal{F}:|F|=i\}|$. Many problems in extremal finite set theory ask for the largest size of a family in a class $\mathbb{A} \subseteq 2^{2^{[n]}}$. This question is equivalent to determining $\max _{\mathcal{F} \in \mathbb{A}} \alpha(\mathcal{F}) \cdot \mathbf{1}$, where $\mathbf{1}$ is the vector of length $n+1$ with all entries being 1 , and $\cdot$ denotes the scalar product.

More generally, consider a weight function $w:\{0,1, \ldots, n\} \rightarrow \mathbb{R}$, and assume we want to maximize $w(\mathcal{F}):=\sum_{F \in \mathcal{F}} w(|F|)$. Then this is equivalent to maximizing $\alpha(\mathcal{F}) \cdot \mathbf{w}$, where $\mathbf{w}=(w(0), w(1), \ldots, w(n))$. As $\mathbb{A} \subseteq 2^{2^{[n]}}$ holds, we have $\{\alpha(\mathcal{F}): \mathcal{F} \in \mathbb{A}\} \subseteq \mathbb{R}^{n+1}$ and therefore we can consider its convex hull $\mu(\mathbb{A})$, that we call the profile polytope of $\mathbb{A}$. It is well known that any weight function with the above property is maximized by an extreme point of $\mu(\mathbb{A})$ (a point that is not a convex combination of other points of $\mu(\mathbb{A})$ ), moreover if such a weight function is non-negative, then it is maximized by an essential extreme point, i.e. an extreme point which is maximal with respect to the coordinate-wise ordering. First results concerning profile polytopes were obtained in [8-11,20] and the not too recent monograph of Engel [7] contains a chapter devoted to this topic.

Using this we can determine $\operatorname{La}\left(n, P_{3}, P_{2}\right)$, and using induction with this as the base case, one can determine $\operatorname{La}\left(n, P_{k+1}, P_{k}\right)$, but in other cases we will need a more powerful tool than ordinary profile vectors. The notion of $l$-chain profile vector $\alpha_{l}(\mathcal{F})$ of a family $\mathcal{F} \subseteq 2^{[n]}$ was introduced by Gerbner and Patkós [13] and denotes a vector of length $\left(\begin{array}{c}n+1 \\ l\end{array}\right)$. The coordinates are indexed by $l$-tuples of $[0, n]$, and $\alpha_{l}(\mathcal{F})\left(i_{1}, i_{2}, \ldots, i_{l}\right)$ is the number of chains $F_{1} \subsetneq F_{2} \subsetneq \cdots \subsetneq F_{l}$ such that $F_{j} \in \mathcal{F}$ and $\left|F_{j}\right|=i_{j}$ for all $1 \leq j \leq l$. For a set $\mathbb{A} \subseteq 2^{2^{[n]}}$ one can define the $l$-chain profile polytope $\mu_{l}(\mathbb{A})$, its extreme points and essential extreme points analogously to the above. Note that for $l=1$ we get back the definition of the original profile polytope.

Here we introduce two new types of profile vectors. For a family $\mathcal{F} \subseteq 2^{[n]}$ of sets, let $\beta^{r}(\mathcal{F})=\left(\beta_{0}^{r}, \beta_{1}^{r}, \ldots, \beta_{n-1}^{r}\right)$ denote the $r$-intersection profile vector of $\mathcal{F}$, where $\beta_{i}^{r}=$ $\beta_{i}^{r}(\mathcal{F})=\mid\left\{\left\{F_{1}, F_{2}, \ldots, F_{r}\right\}: F_{j} \in \mathcal{F}\right.$, these are $r$ different sets, and $\left.\left|\bigcap_{j=1}^{r} F_{j}\right|=i\right\} \mid$.

For a family $\mathcal{F} \subseteq 2^{[n]}$ of sets, let $\gamma^{r}(\mathcal{F})=\left(\gamma_{0,1}^{r}, \gamma_{0,2}^{r}, \ldots, \gamma_{0, n}^{r}, \gamma_{1,2}^{r}, \ldots, \gamma_{n-1, n}^{r}\right)$ denote the $r$-intersection-union profile vector of $\mathcal{F}$, where $\gamma_{i, j}^{r}=\gamma_{i, j}^{r}(\mathcal{F})=\mid\left\{\left\{F_{1}, \ldots, F_{r}\right\}\right.$ : $F_{1}, \ldots, F_{r} \in \mathcal{F}$, these are $r$ different sets, $\left|F_{1} \cap \cdots \cap F_{r}\right|=i$, and $\left.\left|F_{1} \cup \cdots \cup F_{r}\right|=j\right\} \mid$. Note that if $\mathcal{A} \subseteq 2^{[n]}$ is an antichain, then $\gamma_{i, j}^{r}(\mathcal{A})>0$ implies $j-i \geq 2$, therefore the number of non-zero coordinates in $\gamma^{r}(\mathcal{A})$ is at most $\left(\begin{array}{c}n+1 \\ 2\end{array}\right)-n=\left(\begin{array}{l}n \\ 2\end{array}\right) \leq n^{2}$. 
Let us illustrate with two examples why these profile vectors can be useful in counting copies of different posets. Let $K_{r_{1}, r_{2}, \ldots, r_{s}}$ denote the complete $s$-level poset on $\sum_{i=1}^{s} r_{i}$ elements

$$
a_{1}^{1}, a_{2}^{1}, \ldots, a_{r_{1}}^{1}, a_{1}^{2}, a_{2}^{2}, \ldots, a_{r_{2}}^{2}, \ldots, a_{1}^{s}, a_{2}^{s}, \ldots, a_{r_{s}}^{s}
$$

with $a_{h}^{i}<a_{l}^{j}$ if and only if $i<j$. Observe that $\bigvee_{r}=K_{1, r}, B=K_{2,2}$ and $D_{r}=K_{1, r, 1}$.

Let $\mathcal{F}$ be a $P_{3}$-free and $\mathcal{G}$ be a $P_{4}$-free family. We will estimate $c\left(K_{p, r}, \mathcal{F}\right)$ and $c\left(K_{p, r, s}, \mathcal{G}\right)$. If we consider the canonical partitions of $\mathcal{F}=\mathcal{F}_{1} \cup \mathcal{F}_{2}$ and $\mathcal{G}=\mathcal{G}_{1} \cup \mathcal{G}_{2} \cup \mathcal{G}_{3}$, then a copy of $K_{p, r}$ in $\mathcal{F}$ contains $p$ sets from $\mathcal{F}_{1}$ and $r$ sets from $\mathcal{F}_{2}$. If we fix $F_{1}, \ldots, F_{r} \in$ $\mathcal{F}_{2}$, then the $p$ "bottom" sets of the copies of $K_{p, r}$ in $\mathcal{F}$ containing $F_{1}, \ldots, F_{r}$ form an antichain in $\left\{F \in \mathcal{F}_{1}: F \subseteq \bigcap_{j=1}^{r} F_{j}\right\}$. Therefore, by Theorem 1.1, the number of these copies is at most $\left(\begin{array}{c}\left\lfloor\cap_{j=1}^{\mid} \cap_{j=1}^{r} F_{j} \mid / 2\right\rfloor \\ { }_{p}\end{array}\right)$, so summing up for all possible $r$-tuples of $\mathcal{F}_{2}$ we obtain $c\left(K_{p, r}, \mathcal{F}\right) \leq \beta^{r}(\mathcal{F}) \cdot \mathbf{w}_{p}$, and consequently

$$
\operatorname{La}\left(n, P_{3}, K_{p, r}\right) \leq \max \left\{\beta^{r}(\mathcal{A}) \cdot \mathbf{w}_{p}: \mathcal{A} \subseteq 2^{[n]} \text { is an antichain }\right\},
$$

where the $j$ th entry of the vector $\mathbf{w}_{p}$ is $\left(\begin{array}{c}\left({ }^{j} / 2\right\rfloor \\ p\end{array}\right)$.

Similarly, if we consider the canonical partition of $\mathcal{G}=\mathcal{G}_{1} \cup \mathcal{G}_{2} \cup \mathcal{G}_{3}$, then a copy of $K_{p, r, s}$ in $\mathcal{G}$ contains $p$ sets from $\mathcal{G}_{1}, r$ sets from $\mathcal{G}_{2}$ and $s$ sets from $\mathcal{G}_{3}$. If we fix $G_{1}, \ldots, G_{r} \in \mathcal{G}_{2}$, then the bottom $p$ and top $s$ sets of copies of $K_{p, r, s}$ containing $G_{1}, \ldots, G_{r}$ form antichains in $\left\{G \in \mathcal{G}_{1}: G \subseteq \bigcap_{j=1}^{r} G_{j}\right\}$ and $\left\{G \in \mathcal{G}_{3}: G \supseteq \bigcup_{j=1}^{r} G_{j}\right\}$. Therefore, using again Theorem 1.1 , we obtain $c\left(K_{p, r, s}, \mathcal{G}\right) \leq \gamma^{r}\left(\mathcal{G}_{2}\right) \cdot \mathbf{w}_{p, s}$ and consequently

$$
\operatorname{La}\left(n, P_{4}, K_{p, r, s}\right) \leq \max \left\{\gamma^{r}(\mathcal{A}) \cdot \mathbf{w}_{p, s}: \mathcal{A} \subseteq 2^{[n]} \text { is an antichain }\right\}
$$

where the $(i, j)$ th entry of the vector $\mathbf{w}_{p, s}$ is $\left(\begin{array}{c}\left(\begin{array}{c}i \\ i / 2\rfloor \\ p\end{array}\right) \\ \text { (n) }\end{array}\right)\left(\begin{array}{c}\left(\begin{array}{c}n-j \\ (n-j) / 2\rfloor\end{array}\right) \\ s\end{array}\right)$. Therefore determining the convex hull (and more importantly its extreme points) of the $r$-intersection profile vectors and $r$-intersection-union profile vectors of antichains would yield upper bounds on $L a$ functions of complete multi-level posets.

We are not able to determine these convex hulls, we will only obtain upper bounds on the coordinates of these profile vectors.

Theorem 1.8 (a) For every $r \geq 3$ and antichain $\mathcal{A} \subseteq 2^{[n]}, \gamma_{0, n}^{r}(\mathcal{A}) \leq n^{2 r} \gamma_{0, n}^{r}\left(\left(\begin{array}{c}{[n]} \\ \lfloor n / 2\rfloor\end{array}\right)\right)$. If $r=2$ and $n$ is even, then $\gamma_{0, n}^{2}(\mathcal{A}) \leq \gamma_{0, n}^{2}\left(\left(\begin{array}{l}{[n]} \\ n / 2\end{array}\right)\right)$, while if $r=2$ and $n$ is odd, then $\gamma_{0, n}^{2}(\mathcal{A}) \leq\left(\begin{array}{c}n-1 \\ \lfloor n / 2\rfloor-1\end{array}\right)$.

(b) For every $r$ there exists a sequence $l_{n}$ such that if $\mathcal{A} \subseteq 2^{[n]}$ is an antichain, then $\beta_{0}^{r}(\mathcal{A}) \leq n^{2 r+1} \beta_{0}^{r}\left(\left(\begin{array}{c}{[n]} \\ l_{n}\end{array}\right)\right)$.

(c) If $\mathcal{A} \subseteq 2^{[n]}$ is an antichain, then $\beta_{0}^{2}(\mathcal{A}) \leq \frac{1}{2}\left(\begin{array}{c}n \\ \lfloor n / 3\rfloor\end{array}\right)\left(\begin{array}{c}{[2 n / 3\rceil} \\ \lfloor n / 3\rfloor\end{array}\right)$ and this is sharp as shown by $\left(\begin{array}{c}{[n]} \\ \lfloor n / 3\rfloor\end{array}\right)$ if $n \equiv 0,1 \bmod 3$ and by $\left(\begin{array}{c}{[n]} \\ {[n / 3\rceil}\end{array}\right)$ if $n \equiv 2 \bmod 3$.

The $r=2$ part of Theorem 1.8 (a) was proved by Bollobás [2]. Using the above theorem, we can give bounds on $\operatorname{La}\left(n, P_{l\left(Q_{1} \otimes_{r} Q_{2}\right)+1}, Q_{1} \otimes_{r} Q_{2}\right)$.

Theorem 1.9 Let $Q_{1}, Q_{2}$ be two non-empty posets. 
(a) If $r \geq 2$, then we have

$$
\begin{aligned}
& \operatorname{La}\left(n, P_{l\left(Q_{1}\right)+l\left(Q_{2}\right)+1}, Q_{1} \otimes_{r} Q_{2}\right) \leq \\
& n^{2 r+2} \max _{0 \leq i<j \leq n}\left\{\left(\begin{array}{c}
n \\
j
\end{array}\right)\left(\begin{array}{l}
j \\
i
\end{array}\right) \gamma_{0, j-i}^{r}\left(\left(\begin{array}{c}
{[j-i]} \\
\lfloor(j-i) / 2\rfloor
\end{array}\right)\right) L a\left(i, P_{l\left(Q_{1}\right)+1}, Q_{1}\right) L a\left(n-j, P_{l\left(Q_{2}\right)+1}, Q_{2}\right)\right\} .
\end{aligned}
$$

Furthermore, if $r \geq 3$ and Conjecture 1.5 almost holds for the pairs $P_{l\left(Q_{1}\right)+1}, Q_{1}$ and $P_{l\left(Q_{2}\right)+1}, Q_{2}$, then so it does for the pair $P_{l\left(Q_{1} \otimes_{r} Q_{2}\right)+1}, Q_{1} \otimes_{r} Q_{2}$.

(b) If $r=1$, then we have

$$
L a\left(n, P_{l\left(Q_{1}\right)+l\left(Q_{2}\right)+1}, Q_{1} \otimes_{1} Q_{2}\right) \leq \max _{0 \leq j \leq n}\left\{\left(\begin{array}{c}
n \\
j
\end{array}\right) L a\left(j, P_{l\left(Q_{1}\right)+1}, Q_{1}\right) L a\left(n-j, P_{l\left(Q_{2}\right)+1}, Q_{2}\right)\right\} .
$$

Furthermore, if Conjecture 1.5 strongly/almost holds for the pairs $P_{l\left(Q_{1}\right)+1}, Q_{1}$ and $P_{l\left(Q_{2}\right)+1}, Q_{2}$, then so it does for the pair $P_{l\left(Q_{1} \otimes_{1} Q_{2}\right)+1}, Q_{1} \otimes_{1} Q_{2}$.

Theorem 1.10 Let $Q$ be a non-empty poset.

(a) If $r \geq 2$ and $n \in \mathbb{N}$, then there exists an $i=i(r, n)$ such that

$$
L a\left(n, P_{l(Q)+2}, Q \oplus r\right) \leq \max _{0 \leq j \leq n}\left\{\left(\begin{array}{c}
n \\
i
\end{array}\right) \beta_{0}^{r}\left(\left(\begin{array}{c}
{[n-i]} \\
j-i
\end{array}\right)\right) L a\left(j, P_{l(Q)+1}, Q\right)\right\} .
$$

Furthermore, if Conjecture 1.5 almost holds for the pair $P_{l(Q)+1}, Q$, then so it does for the pair $P_{l(Q)+2}, Q_{1} \oplus r$.

(b) If $r=1$, then we have

$$
L a\left(n, P_{l(Q)+2}, Q \oplus 1\right) \leq \max _{0 \leq j \leq n}\left\{\left(\begin{array}{l}
n \\
j
\end{array}\right) L a\left(j, P_{l(Q)+1}, Q\right)\right\} .
$$

Furthermore, if Conjecture 1.5 strongly/almost holds for the pair $P_{l(Q)+1}, Q$, then so it does for the pair $P_{l(Q)+2}, Q \oplus 1$.

We can apply Theorem 1.9 and Theorem 1.10 to complete multi-level posets (Fig. 2).

Corollary 1.11 For any complete multi-level poset $K_{r_{1}, r_{2}, \ldots, r_{s}}$, Conjecture 1.5 almost holds for the pair $P_{s+1}, K_{r_{1}, r_{2}, \ldots, r_{s}}$.

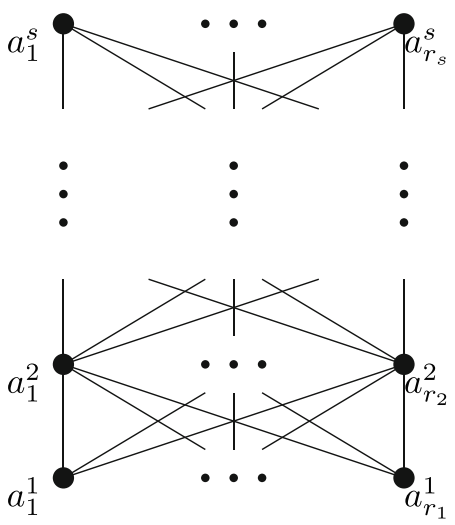

$K_{r_{1}, r_{2}, \ldots, r_{s}}$

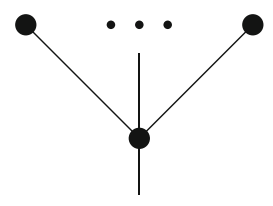

$\bullet$

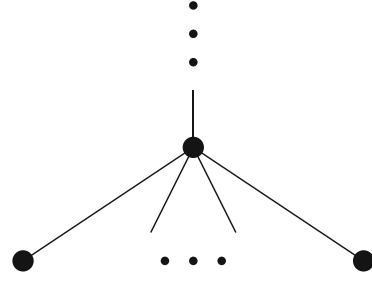

$K_{r, 1,1, \ldots, 1, s}$

Fig. 2 The Hasse diagrams of multi-level posets 
Corollary 1.12 Conjecture 1.5 strongly holds for the pair $P_{s+1}, K_{r_{1}, r_{2}, \ldots, r_{s}}$ if for every $i<s$ at least one of $r_{i}$ and $r_{i+1}$ is equal to 1 .

Corollary 1.12 does not tell us anything about the set sizes in the family containing the most number of copies of $K_{r_{1}, r_{2}, \ldots, r_{s}}$. The next theorem gives more insight for an even more special case.

Theorem 1.13 The value of $\operatorname{La}\left(n, P_{l+3}, K_{r, 1, \ldots, 1, s}\right)$ (where there are $l$ copies of 1 in $\left.K_{r, 1, \ldots, 1, s}\right)$ is attained for a family $\mathcal{F}=\cup_{j=1}^{l+2}\left(\begin{array}{c}{[n]} \\ i_{j}\end{array}\right)$, where $i_{1}=\left\lfloor i_{2} / 2\right\rfloor, i_{l+2}=\left\lfloor\left(n+i_{l+1}\right) / 2\right\rfloor$ and $i_{3}-i_{2}, i_{4}-i_{3}, \ldots, i_{l+1}-i_{l}$ differ by at most 1 .

Finally observe that Conjecture 1.2, if true, and Theorem 1.1 would have another consequence: the asymptotics of $\operatorname{La}(n, P)=\operatorname{La}\left(n, P, P_{1}\right)$ would equal that of $\operatorname{La}\left(n, P_{j}\right)$ for some integer $j$. This is not true in our more general context in the following stronger sense that even the order of magnitude of $L a(n, P, Q)$ can be different from any function $\operatorname{La}\left(n, P_{j}, Q\right)$.

Theorem 1.14 There does not exist any integer $j$ with $\operatorname{La}\left(n, B, P_{2}\right)=\Theta\left(\operatorname{La}\left(n, P_{j}, P_{2}\right)\right)$.

The rest of the paper is organized as follows: we prove Theorem 1.4 and Theorem 1.6 in Section 2. We prove Theorem 1.7 in Section 3. Theorem 1.8, Theorem 1.9, Theorem 1.10, and their corollaries are proved in Section 4, Theorem 1.14 in Section 5, while Section 6 contains some concluding remarks.

\section{Proofs of Theorem 1.4 and Theorem 1.6}

Proof of Theorem 1.4 We start by proving the upper bound. Let $\mathcal{F} \subseteq 2^{[n]}$ be a $D_{k}$-free family. As for any poset $P$, the canonical partition of a $P$-free family can consist of at most $|P|-1$ antichains, we can assume that the canonical partition of $\mathcal{F}$ is $\bigcup_{i=1}^{k+1} \mathcal{F}_{i}$. In any copy of $D_{l}$ in $\mathcal{F}$, the sets corresponding to the top and bottom element of $D_{l}$ come from $\mathcal{F}_{i}$ and $\mathcal{F}_{j}$ with $i-j \geq 2$. The number of such pairs of indices is $\left(\begin{array}{c}k+1 \\ 2\end{array}\right)-k$. Let us bound the number of copies of $D_{l}$ with top element from $\mathcal{F}_{i}$ and bottom element from $\mathcal{F}_{j}$. As $\mathcal{F}_{i} \cup \mathcal{F}_{j}$ is $P_{3}$-free, there are at most $\operatorname{La}\left(n, P_{3}, P_{2}\right)$ many ways to choose the top and the bottom elements $F_{B} \subset F_{T}$. As $\mathcal{F}$ is $D_{k}$-free, there can be at most $k-1$ sets in $\mathcal{F}$ lying between $F_{B}$ and $F_{T}$, so the number of copies of $D_{l}$ with $F_{B}, F_{T}$ being top and bottom is at most $\left(\begin{array}{c}k-1 \\ l\end{array}\right)$. The upper bound on $\operatorname{La}\left(n, D_{k}, D_{l}\right)$ follows.

For the lower bound we need a construction. Let $\mathcal{F}_{1} \cup \mathcal{F}_{2} \subseteq 2^{[n-k+1]}$ be the canonical partition of the $P_{3}$-free family $\mathcal{F}$ with $c\left(P_{2}, \mathcal{F}\right)=L a\left(n-k+1, P_{3}, P_{2}\right)$. For $j=3,4, \ldots, k+1$ let $\mathcal{F}_{j}=\left\{F \cup[n-k+2, n-k+j-1]: F \in \mathcal{F}_{2}\right\}$, where for $i \leq j,[i, j]$ denotes the set $\{i, i+1, \ldots, j-1, j\}$. We claim that $\mathcal{G}=\cup_{i=1}^{k+1} \mathcal{F}_{i}$ is $D_{k^{-}}$ free with $c\left(D_{l}, \mathcal{F}\right) \geq\left(\begin{array}{c}k-1 \\ l\end{array}\right) L a\left(n-k+1, P_{3}, P_{2}\right)$. Indeed, every set $G \in \mathcal{G}$ is contained in a set $F_{k+1} \in \mathcal{F}_{k+1}$ and contains a set $F_{1} \in \mathcal{F}_{1}$, therefore if there was a copy of $D_{k}$, we could assume that its bottom element is from $\mathcal{F}_{1}$ and its top element is from $\mathcal{F}_{k+1}$. But any $F_{k+1} \in \mathcal{F}_{k+1}$ contains exactly one element from each $\mathcal{F}_{i}$ where $i=2,3, \ldots, k$, so there is no space for a copy of $D_{k}$. On the other hand, for every pair $F_{1} \subset F_{2}$ in $\mathcal{F}_{1} \cup \mathcal{F}_{2}$ we can add $l$ sets from $\left\{F_{2} \cup[n-k+2, n-k+j-1]: j=3,4, \ldots, k+1\right\}$ to form a copy of $D_{l}$. For each such pair we will obtain $\left(\begin{array}{c}k-1 \\ l\end{array}\right)$ such copies. 
Proof of Theorem 1.6 To prove (a), by symmetry, it is enough to show $\operatorname{La}\left(n, \bigvee, P_{2}\right)=$ $\left(\begin{array}{c}n \\ \lfloor n / 2\rfloor\end{array}\right)$. Consider any $\bigvee$-free family $\mathcal{F} \subseteq 2^{[n]}$. As the $\bigvee$-free property implies the $P_{3}$-free property, the canonical partition of $\mathcal{F}$ is $\mathcal{F}_{1} \cup \mathcal{F}_{2}$. By the $\bigvee$-free property of $\mathcal{F}$, elements of $\mathcal{F}_{1}$ are contained in at most one copy of $P_{2}$. Also, every copy of $P_{2}$ in $\mathcal{F}$ must contain a set from $\mathcal{F}_{1}$. Sperner's theorem yields $c\left(P_{2}, \mathcal{F}\right) \leq\left|\mathcal{F}_{1}\right| \leq\left(\begin{array}{c}n \\ \lfloor n / 2\rfloor\end{array}\right)$. On the other hand $\mathcal{F}:=\{F \subseteq[n]:|F|=\lfloor n / 2\rfloor\} \cup\{[n]\}$ is $\bigvee$-free and every $\lfloor n / 2\rfloor$-element set forms a copy of $P_{2}$ with $[n]$.

We continue with proving (b). We will need the following definition. For any family $\mathcal{F}$, the comparability graph of $\mathcal{F}$ has vertex set $\mathcal{F}$ and two sets $F, F^{\prime} \in \mathcal{F}$ are joined by an edge if $F \subseteq F^{\prime}$ or $F^{\prime} \subseteq F$ holds. The connected components of the comparability graph of $\mathcal{F}$ are said to be the components of $\mathcal{F}$. If a family $\mathcal{F}$ is both $\bigvee$-free and $\bigwedge$ free, then its components are either isolated vertices or isolated edges in the comparability graph. Therefore $c\left(P_{2}, \mathcal{F}\right)$ is the number of components that are isolated edges. It follows that $\operatorname{La}\left(n,\{\bigvee, \bigwedge\}, P_{2}\right) \leq \frac{1}{2} \operatorname{La}\left(n,\{\bigvee, \bigwedge\}, P_{1}\right)=\left(\begin{array}{c}n-1 \\ \lfloor(n-1) / 2\rfloor\end{array}\right)$ where the result in the last equation was proved by Katona and Tarján [21]. The construction (given also in [21]) $\mathcal{F}:=$ $\left(\begin{array}{c}{[n-1]} \\ \lfloor(n-1) / 2\rfloor\end{array}\right) \cup\left\{\{n\} \cup F: F \in\left(\begin{array}{c}{[n-1]} \\ \lfloor(n-1) / 2\rfloor\end{array}\right)\right\}$ shows that the above upper bound can be attained.

To prove (c), let us consider a $B$-free family $\mathcal{F} \subseteq 2^{[n]}$ and let $\mathcal{M}=\left\{M \in \mathcal{F}: \exists F^{\prime}, F^{\prime \prime} \in\right.$ $\mathcal{F}$ such that $\left.F^{\prime} \subsetneq M \subsetneq F^{\prime \prime}\right\}$. As $B$-free implies $P_{4}$-free, we obtain that $\mathcal{M}$ is an antichain, thus $|\mathcal{M}| \leq\left(\begin{array}{c}n \\ \lfloor n / 2\rfloor\end{array}\right)$, by Theorem 1.1. Moreover, if $M \in \mathcal{M}$, then there do not exist two elements $F_{1}, F_{2} \in \mathcal{F}$ with $M \subsetneq F_{1}, F_{2}$. Indeed, by the definition of $\mathcal{M}$ there exists $F^{\prime} \in \mathcal{F}$ with $F^{\prime} \subsetneq M$, and $F^{\prime}, M, F_{1}, F_{2}$ would form a copy of $B$. Similarly, for every $M \in \mathcal{M}$ there exists exactly one element $F \in \mathcal{F}$ with $F \subsetneq M$. Therefore, a copy of $D_{r}$ contains $r$ elements of $M$, and they determine the remaining two elements, which implies $c\left(D_{r}, \mathcal{F}\right) \leq$ $\left(\begin{array}{c}|\mathcal{M}| \\ r\end{array}\right) \leq\left(\begin{array}{c}\left(\begin{array}{c}n \\ \lfloor n / 2\end{array}\right) \\ r\end{array}\right)$. The construction $\mathcal{F}:=\{\emptyset,[n]\} \cup\left(\begin{array}{c}{[n]} \\ \lfloor n / 2\rfloor\end{array}\right)$ shows that this upper bound can be attained.

To prove (d), by symmetry, it is enough to show $\operatorname{La}\left(n, \bigvee, \bigwedge_{r}\right)=\left(\begin{array}{c}\left(\begin{array}{c}n \\ \lfloor n / 2\rfloor\end{array}\right) \\ r\end{array}\right)$. If $\mathcal{F}$ is $\bigvee$-free, then it is in particular $P_{3}$-free. Consider its canonical partition $\mathcal{F}^{r}=\mathcal{F}_{1} \cup \mathcal{F}_{2}$. Then a copy of $\bigwedge_{r}$ contains $r$ elements from $\mathcal{F}_{1}$ and one from $\mathcal{F}_{2}$. Moreover, an $r$-tuple from $\mathcal{F}_{1}$ may form a copy of $\bigwedge_{r}$ with at most one element from $\mathcal{F}_{2}$, otherwise there is a copy (actually $r$ copies) of $\bigvee$ in $\mathcal{F}$. As $\mathcal{F}_{1}$ is an antichain, by Theorem 1.1, the upper bound $\operatorname{La}\left(n, \bigvee, \bigwedge_{r}\right) \leq\left(\begin{array}{c}\left(\begin{array}{c}n \\ \lfloor n / 2\rfloor\end{array}\right) \\ r\end{array}\right)$ follows and $\mathcal{F}:=\{[n]\} \cup\left(\begin{array}{c}{[n]} \\ \lfloor n / 2\rfloor\end{array}\right)$ shows that this can be attained.

\section{The profile polytope method}

In this section we prove Theorem 1.7 after stating a result concerning $l$-chain profile vectors, due to Gerbner and Patkós [13].

Let $\mathbb{S}_{n, k}$ be the class of all $k$-Sperner (i.e. $P_{k+1}$-free) families on $[n]$.

Lemma 3.1 (Gerbner, Patkós, [13]) The essential extreme points of $\mu_{l}\left(\mathbb{S}_{n, k}\right)$ are the l-chain vectors of $k$-Sperner families that consist of the union of $k$ full levels.

Let us state the immediate consequence of the above lemma that we will use in our proofs in the remainder of this section. 
Corollary 3.2 Let $l \leq k$ and $w:\left(\begin{array}{c}2^{[n]} \\ l\end{array}\right) \rightarrow \mathbb{R}^{+}$be a weight function such that $w\left(\left\{F_{1}, F_{2}, \ldots F_{l}\right\}\right)$ depends only on $\left|F_{1}\right|,\left|F_{2}\right|, \ldots,\left|F_{l}\right|$. Then the maximum of

$$
\sum_{F_{1} \subsetneq F_{2} \subsetneq \cdots \subsetneq F_{l}, F_{i} \in \mathcal{F}} w\left(\left\{F_{1}, F_{2}, \ldots, F_{l}\right\}\right)
$$

over all families $\mathcal{F} \in \mathbb{S}_{n, k}$ is attained at some family that consists of $k$ full levels.

Proof of Theorem 1.7. To prove (a), we show $\operatorname{La}\left(n, P_{3}, \bigwedge_{r}\right)=\left(\begin{array}{l}n \\ i_{r}\end{array}\right)\left(\begin{array}{c}\left(\begin{array}{c}i_{r} \\ \left.i_{r} / 2\right\rfloor\end{array}\right) \\ r\end{array}\right)$ as the other statement follows by symmetry. Let us consider the canonical partition $\mathcal{F}_{1} \cup \mathcal{F}_{2}$ of a $P_{3}$-free family $\mathcal{F}$. Note that a copy of $\bigwedge_{r}$ contains exactly one element $F$ from $\mathcal{F}_{2}$ and $r$ elements $F_{1}, F_{2}, \ldots, F_{r} \in \mathcal{F}_{1}$ with $F_{i} \subsetneq F$ for all $1,2, \ldots, r$. Let us consider a set $F \in \mathcal{F}_{2}$. The sets of $\mathcal{F}_{1}$ contained in $F$ form an antichain, thus by Theorem 1.1, their number is at most $\left(\begin{array}{c}|F| \\ \lfloor|F| / 2\rfloor\end{array}\right)$. Therefore, the number of copies of $\bigwedge_{r}$ that contain $F$ is at most $\left.\left({ }_{\lfloor|F| / 2\rfloor}^{|F|}\right)\right)$ and we obtain

$$
c\left(\bigwedge_{r}, \mathcal{F}\right) \leq \sum_{F \in \mathcal{F}_{2}}\left(\begin{array}{c}
\left(\begin{array}{c}
|F| \\
\lfloor|F| / 2\rfloor \\
r
\end{array}\right)
\end{array}\right) \leq \max _{\mathcal{A} \in \mathbb{S}_{n, 1}} \sum_{A \in \mathcal{A}}\left(\begin{array}{c}
\left(\begin{array}{c}
|A| \\
\lfloor|| / 2\rfloor \\
r
\end{array}\right)
\end{array}\right) .
$$

Therefore, if we set $w(i):=\left({ }_{(i / 2\rfloor}^{i}\right)$, then we can apply Corollary 3.2 with $l=k=1$ to obtain $c\left(\bigwedge_{r}, \mathcal{F}\right) \leq \max _{0 \leq i \leq n}\left(\begin{array}{c}n \\ i\end{array}\right) w(i)$. On the other hand, the families $\mathcal{F}(i)=\left(\begin{array}{c}{[n]} \\ i\end{array}\right) \cup\left(\begin{array}{c}{[n]} \\ \lfloor i / 2\rfloor\end{array}\right)$ are $P_{3}$-free and $c\left(\bigwedge_{r}, \mathcal{F}(i)\right)=\left(\begin{array}{l}n \\ i\end{array}\right) w(i)$ showing $L a\left(n, P_{3}, \bigwedge_{r}\right)=\max _{0 \leq i \leq n}\left(\begin{array}{c}n \\ i\end{array}\right) w(i)$. To obtain the value of $i_{r}$ we need to maximize $f(i):=\left(\begin{array}{c}n \\ i\end{array}\right) w(i)$. We have

$$
\frac{f(i)}{f(i+1)}=\frac{i+1}{n-i} \cdot \frac{\prod_{j=0}^{r-1}\left(\left(\begin{array}{c}
i \\
\lfloor i / 2\rfloor
\end{array}\right)-j\right)}{\prod_{j=0}^{r-1}\left(\left(\begin{array}{c}
i+1 \\
\lfloor(i+1) / 2\rfloor
\end{array}\right)-j\right)}=(1+o(1)) \frac{i+1}{2^{r}(n-i)}
$$

when $i$ tends to infinity with $n$. For constant values of $i$, the ratio $f(i) / f(i+1)$ is easily seen to be smaller than 1 (if $n$ is big enough), therefore the maximum of $f(i)$ is attained at $i_{r}=(1+o(1)) \frac{2^{r}}{2^{r}+1} n$ as stated.

To prove (b), we consider the canonical partition of a $P_{4}$-free family $\mathcal{F} \subseteq 2^{[n]}$. Any copy of $D_{r}$ in $\mathcal{F}$ must contain one set from $\mathcal{F}_{1}, \mathcal{F}_{3}$ each and $r$ sets from $\mathcal{F}_{2}$. For any $F_{1} \in \mathcal{F}_{1}, F_{3} \in \mathcal{F}_{3}$ with $F_{1} \subset F_{3}$, the number of copies of $D_{r}$ containing $F_{1}$ and $F_{3}$ is $\left(\begin{array}{c}m \\ r\end{array}\right)$, where $m=\left|\mathcal{M}_{F_{1}, F_{3}}\right|$ with $\mathcal{M}_{F_{1}, F_{3}}=\left\{F \in \mathcal{F}_{2}: F_{1} \subset F \subset F_{3}\right\}$. As $\mathcal{M}_{F_{1}, F_{3}}^{\prime}=\left\{M \backslash F_{1}\right.$ : $\left.M \in \mathcal{M}_{F_{1}, F_{3}}\right\}$ is an antichain in $F_{3} \backslash F_{1}$, we have $m \leq\left(\begin{array}{c}\left|F_{3}\right|-\left|F_{1}\right| \\ \left\lfloor\left(\left|F_{3}\right|-\left|F_{1}\right|\right) / 2\right\rfloor\end{array}\right)$. Therefore, we obtain

$$
c\left(D_{r}, \mathcal{F}\right) \leq \sum_{F_{1} \in \mathcal{F}_{1}, F_{3} \in \mathcal{F}_{3}, F_{1} \subset F_{3}}\left(\begin{array}{c}
\left(\begin{array}{c}
\left|F_{3}\right|-\left|F_{1}\right| \\
\left\lfloor\left(\left|F_{3}\right|-\left|F_{1}\right|\right) / 2\right\rfloor \\
r
\end{array}\right) \\
r
\end{array}\right) \leq \max _{0 \leq i<j \leq n}\left(\begin{array}{c}
n \\
j
\end{array}\right)\left(\begin{array}{c}
j \\
i
\end{array}\right)\left(\begin{array}{c}
j-i \\
\lfloor(j-i) / 2\rfloor \\
r
\end{array}\right),
$$

where to obtain the last inequality we applied Corollary 3.2 with $l=k=2$ and $w(i, j)=$ $\left(\begin{array}{c}\left.\left(\begin{array}{c}j-i \\ r\end{array}\right) / 2\right\rfloor \\ )\end{array}\right)$. Observe that if $i_{r}$ and $j_{r}$ are the values for which this maximum is taken, then for the family $\mathcal{F}=\left(\begin{array}{c}{[n]} \\ i_{r}\end{array}\right) \cup\left(\begin{array}{c}{[n]} \\ j_{r}\end{array}\right) \cup\left(\begin{array}{c}{[n]} \\ \left\lfloor\left(i_{r}+j_{r}\right) / 2\right\rfloor\end{array}\right)$ we have $c\left(D_{r}, \mathcal{F}\right)=\left(\begin{array}{c}n \\ j_{r}\end{array}\right)\left(\begin{array}{c}j_{r} \\ i_{r}\end{array}\right)\left(\begin{array}{c}\left(\begin{array}{c}j_{r}-i_{r} \\ \left.\left.j_{r}-i_{r}\right) / 2\right\rfloor\end{array}\right) \\ r\end{array}\right)$.

To obtain the value of $i_{r}$ and $j_{r}$ let us fix $x=j-i$ first. Note that $\left(\begin{array}{l}n \\ j\end{array}\right)\left(\begin{array}{l}j \\ i\end{array}\right)=\left(\begin{array}{c}n \\ x\end{array}\right)\left(\begin{array}{c}n-x \\ i\end{array}\right)$, so we have

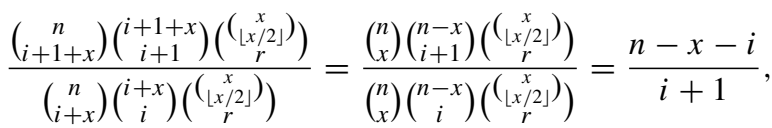

which implies that $i_{r}+j_{r}=n$ or $i_{r}+j_{r}=n-1$ holds. 
Let $g(i)=\left(\begin{array}{c}n \\ i\end{array}\right)\left(\begin{array}{c}n-i \\ i\end{array}\right)\left(\begin{array}{c}\left(\begin{array}{c}n-i \\ (n-i) / 2\rfloor\end{array}\right) \\ r\end{array}\right)$, then

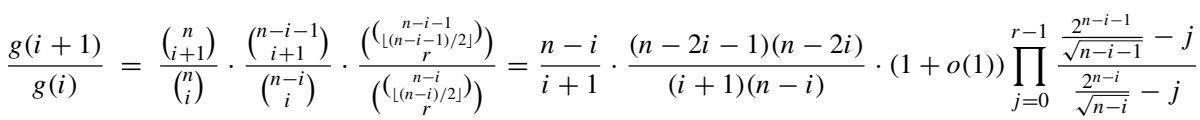

$$
\begin{aligned}
& =(1+o(1)) \frac{(n-2 i-1)(n-2 i)}{(i+1)^{2} 2^{r}},
\end{aligned}
$$

where the second equality uses Stirling's formula and its consequence $\left(\begin{array}{c}N \\ N / 2\end{array}\right)=(\alpha+$ $o(1)) \frac{2^{N}}{\sqrt{N}}$ for some constant $\alpha$. This implies that the maximum of $g(i)$ is attained at $i=(1+o(1)) \frac{n}{2^{r / 2}+2}$.

To prove (c), we again consider the canonical partition of a $P_{3}$-free family $\mathcal{F} \subseteq 2^{[n]}$. A copy of $N$ in $\mathcal{F}$ must contain two sets from $\mathcal{F}_{1}$ and two from $\mathcal{F}_{2}$. Let $a, b, c, d$ be the four elements of $N$ with $a \leq c$ and $b \leq c, d$. For every copy of $N$ in $\mathcal{F}$ there is a bijection $\phi$ from $N$ to that copy. Then we count the copies of $N$ in $\mathcal{F}$ according to the images $\phi(b), \phi(c)$. Clearly, they form a 2-chain in $\mathcal{F}$, the possible images of $d$ form an antichain among those sets of $\mathcal{F}_{2}$ that contain $\phi(b)$ and the possible images of $a$ form an antichain among those sets of $\mathcal{F}_{1}$ that are contained by $\phi(c)$. Therefore, we obtain

$$
c(N, \mathcal{F}) \leq \sum_{F_{1}, F_{2} \in \mathcal{F}, F_{1} \subset F_{2}}\left(\begin{array}{c}
n-\left|F_{1}\right| \\
\left\lfloor\frac{n-\left|F_{1}\right|}{2}\right\rfloor
\end{array}\right)\left(\begin{array}{c}
\left|F_{2}\right| \\
\left\lfloor\frac{\left|F_{2}\right|}{2}\right\rfloor
\end{array}\right) \leq \max _{0 \leq i<j \leq n}\left(\begin{array}{c}
n \\
j
\end{array}\right)\left(\begin{array}{c}
j \\
i
\end{array}\right)\left(\begin{array}{c}
n-i \\
\left\lfloor\frac{n-i}{2}\right\rfloor
\end{array}\right)\left(\begin{array}{c}
j \\
\left\lfloor\frac{j}{2}\right\rfloor
\end{array}\right),
$$

where to obtain the last inequality we applied Corollary 3.2 with $l=k=2$ and $w(i, j)=$ $\left(\begin{array}{c}n-i \\ \left\lfloor\frac{n-i}{2}\right\rfloor\end{array}\right)\left(\begin{array}{c}j \\ \left\lfloor\frac{j}{2}\right\rfloor\end{array}\right)$. We have $\left(\begin{array}{l}n \\ j\end{array}\right)\left(\begin{array}{l}j \\ i\end{array}\right)=\left(\begin{array}{c}n \\ j-i\end{array}\right)\left(\begin{array}{c}n-j+i \\ i\end{array}\right)$, thus we get

$$
c(N, \mathcal{F}) \leq \max _{0 \leq i<j \leq n}\left(\begin{array}{c}
n \\
j-i
\end{array}\right)\left(\begin{array}{c}
n-j+i \\
i
\end{array}\right)\left(\begin{array}{c}
n-i \\
\left\lfloor\frac{n-i}{2}\right\rfloor
\end{array}\right)\left(\begin{array}{c}
j \\
\left\lfloor\frac{j}{2}\right\rfloor
\end{array}\right)=\max _{0 \leq i<j \leq n} o\left(2^{n+n-j+i+n-i+j}\right)=o\left(2^{3 n}\right) .
$$

Note that $j=\lceil 3 n / 4\rceil$ and $i=\lceil n / 4\rceil$ show the exponent cannot be improved with this method.

To obtain the lower bound, consider the $P_{3}$-free families $\mathcal{F}_{i, j}=\left(\begin{array}{c}{[n]} \\ i\end{array}\right) \cup\left(\begin{array}{c}{[n]} \\ j\end{array}\right)$ with $0 \leq$ $i<j \leq n$. Observe that we have $g(i, j):=\frac{1}{4}\left(\begin{array}{l}n \\ j\end{array}\right)\left(\begin{array}{c}j \\ i\end{array}\right)\left(\begin{array}{c}n-i \\ j-i\end{array}\right) \leq c\left(N, \mathcal{F}_{i, j}\right)$ (the $1 / 4$-factor is due to the fact that copies of $B$ are counted 4 times as copies of $N)$. To maximize $g(i, j)$ we first fix $j-i$ and consider

$$
\frac{g(i+1, j+1)}{g(i, j)}=\frac{(n-j)^{2}(j+1)}{(i+1)^{2}(n-i)} .
$$

It is easy to see that this fraction becomes smaller than 1 when $i$ is $n-j+1$. Thus the maximum of $g(i, j)$ is asymptotically achieved when $i=n-j$.

Similarly, we have

$$
\frac{g(n-j-1, j+1)}{g(n-j, j)}=\frac{(j+1)^{2}(n-j)^{4}}{(2 j-n+2)^{3}(2 j-n+1)^{3}} .
$$

Therefore, if we write $j=(c+o(1)) n$, we obtain that $g$ is maximized when $\frac{c^{2}(1-c)^{4}}{(2 c-1)^{6}}=1$. After taking the square root of the expression on the left hand side, this is equivalent to that $0=7 c^{3}-10 c^{2}+5 c-1$ holds. The solution of this equation is $c_{0}=0.69922 \ldots$ As $g(n-j, j)=\frac{1}{4}\left(\begin{array}{c}n \\ j\end{array}\right)\left(\begin{array}{c}j \\ n-j\end{array}\right)^{3}=\Omega\left(2^{h(j / n)+3 \frac{j}{n} h((n-j) / j)} / n^{2}\right)$, the lower bound follows by plugging in $j=0.69922 n$. 


\section{The $\otimes_{r}$ operation and copies of complete multi-level posets}

In this section we prove results concerning the binary operation $Q_{1} \otimes_{r} Q_{2}$. Let us recall the definition of the two new types of profile vectors from the introduction.

For a family $\mathcal{F} \subseteq 2^{[n]}$ of sets, let $\beta^{r}(\mathcal{F})=\left(\beta_{0}^{r}, \beta_{1}^{r}, \ldots, \beta_{n-1}^{r}\right)$ denote the $r$ intersection profile vector of $\mathcal{F}$, where $\beta_{i}^{r}=\beta_{i}^{r}(\mathcal{F})=\mid\left\{\left\{F_{1}, F_{2}, \ldots, F_{r}\right\}: F_{j} \in\right.$ $\mathcal{F}$, these are $r$ different sets and $\left.\left|\bigcap_{j=1}^{r} F_{j}\right|=i\right\} \mid$.

For a family $\mathcal{F} \subseteq 2^{[n]}$ of sets, let $\gamma^{r}(\mathcal{F})=\left(\gamma_{0,1}^{r}, \gamma_{0,2}^{r}, \ldots, \gamma_{0, n}^{r}, \gamma_{1,2}^{r}, \ldots, \gamma_{n-1, n}^{r}\right)$ denote the $r$-intersection-union profile vector of $\mathcal{F}$, where $\gamma_{i, j}^{r}=\gamma_{i, j}^{r}(\mathcal{F})=\mid\left\{\left\{F_{1}, \ldots, F_{r}\right\}\right.$ : $F_{1}, \ldots, F_{r} \in \mathcal{F}$, these are $r$ different sets, $\left.\left|F_{1} \cap \cdots \cap F_{r}\right|=i,\left|F_{1} \cup \cdots \cup F_{r}\right|=j\right\} \mid$. Note that if $\mathcal{A} \subseteq 2^{[n]}$ is an antichain, then $\gamma_{i, j}^{r}(\mathcal{A})>0$ implies $j-i \geq 2$, therefore the number of non-zero coordinates in $\gamma^{r}(\mathcal{A})$ is at most $\left(\begin{array}{c}n+1 \\ 2\end{array}\right)-n=\left(\begin{array}{c}n \\ 2\end{array}\right) \leq n^{2}$.

Note that Theorem 1.8 is about special coordinates, so the next two theorems imply that result.

Theorem 4.1 (a) If $\mathcal{F} \subseteq 2^{[n]}$ is an antichain and $j-i$ is even, then $\gamma_{i, j}^{2}(\mathcal{F}) \leq$ $\gamma_{i, j}^{2}\left(\left(\begin{array}{c}{[n]} \\ (i+j) / 2\end{array}\right)\right)=\frac{1}{2}\left(\begin{array}{c}n \\ j\end{array}\right)\left(\begin{array}{c}j \\ i\end{array}\right)\left(\begin{array}{c}j-i \\ (j-i) / 2\end{array}\right)$. If $j-i$ is odd, then $\gamma_{i, j}^{2}(\mathcal{F}) \leq\left(\begin{array}{c}n \\ j\end{array}\right)\left(\begin{array}{l}j \\ i\end{array}\right)\left(\begin{array}{c}j-i-1 \\ \left.\left(\begin{array}{l}j-i \\ j\end{array}\right) / 2\right\rfloor-1\end{array}\right)$. (b) If $\mathcal{F}$ is an antichain and $r \geq 3$, then $\gamma_{i, j}^{r}(\mathcal{F}) \leq n^{2 r} \gamma_{i, j}^{r}\left(\left(\begin{array}{c}{[n]} \\ \lfloor(i+j) / 2\rfloor\end{array}\right)\right)$.

During the proof we will use several times that the number of pairs $A \subset B \subset[n]$ with $|A|=a,|B|=b$ is $\left(\begin{array}{l}n \\ b\end{array}\right)\left(\begin{array}{l}b \\ a\end{array}\right)=\left(\begin{array}{c}n \\ b-a\end{array}\right)\left(\begin{array}{c}n-(b-a) \\ a\end{array}\right)$. The first calculation is obvious, for the second calculation we pick first $B \backslash A$ from $[n]$ and then $A$ from $[n] \backslash(B \backslash A)$.

Proof To see (a), we first consider the special case $i=0, j=n$. Observe that $\gamma_{0, n}^{2}(\mathcal{F})$ is the number of complement pairs in $\mathcal{F}$. In an antichain, by Theorem 1.1, this is at most $|\mathcal{F}| / 2 \leq\left(\begin{array}{c}n \\ \lfloor n / 2\rfloor\end{array}\right) / 2$. If $n$ is even, then this is achieved when $\mathcal{F}=\left(\begin{array}{l}{[n]} \\ n / 2\end{array}\right)$, while the case of odd $n$ was solved by Bollobás [2], who showed that the number of such pairs is at most $\left(\begin{array}{c}n-1 \\ \lfloor n / 2\rfloor-1\end{array}\right)$, and this is sharp as shown by $\left\{F \in\left(\begin{array}{c}{[n]} \\ \lfloor n / 2\rfloor\end{array}\right): 1 \in F\right\} \cup\left\{F \in\left(\begin{array}{c}{[n]} \\ {[n / 2\rceil}\end{array}\right): 1 \notin F\right\}$.

To see the general statement, observe that for a pair $I \subset J$, writing $\mathcal{F}_{I, J}=\{F \in \mathcal{F}$ :

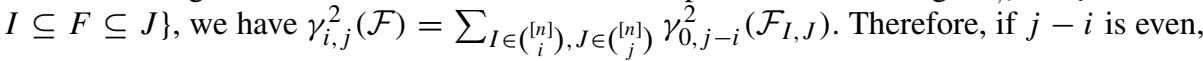
we obtain $\gamma_{i, j}^{2}(\mathcal{F}) \leq\left(\begin{array}{l}n \\ j\end{array}\right)\left(\begin{array}{l}j \\ i\end{array}\right) \gamma_{0, j-i}^{2}\left(\left(\begin{array}{c}{[j-i]} \\ j-i) / 2\end{array}\right)\right)=\gamma_{i, j}^{2}\left(\left(\begin{array}{c}{[n]} \\ (j+i) / 2\end{array}\right)\right)$, while if $j-i$ is odd, we obtain $\gamma_{i, j}^{2}(\mathcal{F}) \leq\left(\begin{array}{l}n \\ j\end{array}\right)\left(\begin{array}{l}j \\ i\end{array}\right)\left(\begin{array}{c}j-i-1 \\ (\lfloor j-i) / 2\rfloor-1\end{array}\right)$.

To show (b), it is enough to prove the statement for $i=0, j=n$. Indeed, $\gamma_{i, j}^{r}(\mathcal{F})=$ $\sum_{I, J} \gamma_{0, j-i}^{r}\left(\mathcal{F}_{I, J}\right) \leq n^{2 r}\left(\begin{array}{c}n \\ j\end{array}\right)\left(\begin{array}{l}j \\ i\end{array}\right) \gamma_{0, j-i}^{r}\left(\left(\begin{array}{c}{[j-i]} \\ \lfloor(j-i) / 2\rfloor\end{array}\right)=n^{2 r} \gamma_{i, j}^{r}\left(\begin{array}{c}{[n]} \\ \lfloor(j+i) / 2\rfloor\end{array}\right)\right.$.

We proceed by induction on $r$. We postpone the proof of the base case $r=3$, as it is similar to, but more involved than the proof of the induction step. Assume the statement holds for $r-1$ and any $i<j$. Let us consider $r-1$ sets $F_{1}, \ldots, F_{r-1}$ of $\mathcal{F}$ and examine which sets can be added to them as $F_{r}$, to get empty intersection and [ $\left.n\right]$ as the union. Let $\mathcal{F}^{\prime}$ be the family of those sets. Let $A=\cap_{l=1}^{r-1} F_{l}$ and $B=\cup_{l=1}^{r-1} F_{l}$ with $a=|A|$ and $b=|B|$. Then members of $\mathcal{F}^{\prime}$ contain the complement of $B$ and do not intersect $A$, and $\mathcal{F}^{\prime}$ is an antichain. If we remove $\bar{B}=[n] \backslash B$ from them, the resulting family is an antichain on an underlying set of size $b-a$. Thus $\mathcal{F}^{\prime}$ has cardinality at most $w(a, b):=\left(\begin{array}{c}b-a \\ \lfloor(b-a) / 2\rfloor\end{array}\right)$. Note that we count every $r$-tuple $F_{1}, \ldots, F_{r}$ exactly $r$ times. It implies

$$
r \gamma_{0, n}^{r}(\mathcal{F}) \leq \sum_{a<b} \gamma_{a, b}^{r-1}(\mathcal{F}) w(a, b)=\gamma^{r-1}(\mathcal{F}) \cdot \mathbf{w} \leq n^{2} \max _{a<b} \gamma_{a, b}^{r-1}(\mathcal{F}) w(a, b) .
$$


By induction this is at most $n^{2} n^{2 r-2} \max _{a<b} \gamma_{a, b}^{r-1}\left(\left(\begin{array}{c}{[n]} \\ \lfloor(a+b) / 2\rfloor\end{array}\right)\right) w(a, b)$. Let

$$
\begin{aligned}
f(a, b) & =\gamma_{a, b}^{r-1}\left(\left(\begin{array}{c}
{[n]} \\
\lfloor(a+b) / 2\rfloor
\end{array}\right)\right) w(a, b) \\
& =\left(\begin{array}{c}
n \\
b-a
\end{array}\right)\left(\begin{array}{c}
n-(b-a) \\
a
\end{array}\right) \gamma_{0, b-a}^{r-1}\left(\left(\begin{array}{c}
{[b-a]} \\
\lfloor(a+b) / 2\rfloor-a
\end{array}\right)\right)\left(\begin{array}{c}
b-a \\
\lfloor(b-a) / 2\rfloor
\end{array}\right) .
\end{aligned}
$$

If we fix $b-a$ and consider $\frac{f(a, b)}{f(a+1, b+1)}=\frac{a+1}{n-(b-a)-a}$, we can see that the maximum is taken when $b+a=n$ or $b+a=n-1$, depending on the parity of $b-a$ and $n$.

Let $a^{*}, b^{*}$ be the values for which the above maximum is taken. Note that for any $a^{*}<$ $p<b^{*}$ we have $r \gamma_{0, n}^{r}\left(\left(\begin{array}{c}{[n]} \\ p\end{array}\right)\right) \geq\left(\begin{array}{c}n \\ b^{*}\end{array}\right)\left(\begin{array}{c}b^{*} \\ a^{*}\end{array}\right) \gamma_{0, b^{*}-a^{*}}^{r-1}\left(\left(\begin{array}{c}\left.b^{*}-a^{*}\right] \\ p-a^{*}\end{array}\right)\right)\left(\begin{array}{c}b^{*}-a^{*} \\ p-n+b^{*}\end{array}\right)$, by counting only those $r$-tuples where the first $r-1$ sets have intersection of size $a^{*}$ and union of size $b^{*}$. (This way we count those $r$-tuples at most $r$ times). This is exactly $f\left(a^{*}, b^{*}\right)$ if $p=\lfloor n / 2\rfloor=$ $\left\lfloor\left(a^{*}+b^{*}\right) / 2\right\rfloor$, so we obtained $r \gamma_{0, n}^{r}(\mathcal{F}) \leq n^{2 r} r \gamma_{0, n}^{r}\left(\left(\begin{array}{c}{[n]} \\ \lfloor n / 2\rfloor\end{array}\right)\right)$ as required.

For $r=3$, we similarly consider two members of $\mathcal{F}$ and examine which sets can be added to them to get empty intersection and $[n]$ as the union. This leads to

$$
3 \gamma_{0, n}^{3}(\mathcal{F}) \leq n^{2} \max _{a<b} \gamma_{a, b}^{2}(\mathcal{F}) w(a, b) .
$$

Note that if the maximum is taken at $a^{\prime}$ and $b^{\prime}$ with $b^{\prime}-a^{\prime}$ even, then part (a) of the theorem gives $\gamma_{a^{\prime}, b^{\prime}}^{2}(\mathcal{F}) \leq \gamma_{a^{\prime}, b^{\prime}}^{2}\left(\left(_{\left(b^{\prime}+a^{\prime}\right) / 2}^{[n]}\right)\right)$, so $3 \gamma_{0, n}^{3}(\mathcal{F}) \leq n^{2} \gamma_{a^{\prime}, b^{\prime}}^{2}\left(\left(_{\left(b^{\prime}+a^{\prime}\right) / 2}^{[n]}\right) w\left(a^{\prime}, b^{\prime}\right)\right.$. This essentially lets us use $r=2$ as the base case of induction, and finish the proof of this case similarly to the induction step above.

Let us choose $a^{*}, b^{*}$ that maximizes this upper bound with $b^{*}-a^{*}=$ $b^{\prime}-a^{\prime}$. Similarly to the computation about $f(a, b)$, we have $a^{*}+b^{*}=$ $n$ or $n-1$ depending on the parity of $n$. Then we obtain $3 \gamma_{0, n}^{3}(\mathcal{F}) \leq$ $n^{2} \gamma_{a^{*}, b^{*}}^{2}\left(\left(\begin{array}{c}{[n]} \\ \left(b^{*}+a^{*}\right) / 2\end{array}\right)\right) w\left(a^{*}, b^{*}\right) \leq n^{2}\left(\begin{array}{c}n \\ b^{*}\end{array}\right)\left(\begin{array}{c}b^{*} \\ a^{*}\end{array}\right) \gamma_{0, b^{*}-a^{*}}^{2}\left(\left(\begin{array}{c}{\left[b^{*}-a^{*}\right]} \\ \left(b^{*}+a^{*}\right) / 2\end{array}\right)\right) w\left(a^{*}, b^{*}\right)$. The lower bound on $3 \gamma_{0, n}^{3}\left(\left(\begin{array}{c}{[n]} \\ \lfloor n / 2\rfloor\end{array}\right)\right)$ is $\left(\begin{array}{c}n \\ b^{*}\end{array}\right)\left(\begin{array}{c}b^{*} \\ a^{*}\end{array}\right) \gamma_{0, b^{*}-a^{*}}^{2}\left(\left(\begin{array}{c}{\left[b^{*}-a^{*}\right]} \\ p-a^{*}\end{array}\right)\right)\left(\begin{array}{c}b^{*}-a^{*} \\ p-a^{*}\end{array}\right)$, as in the inductive step.

However, if $b^{\prime}-a^{\prime}$ is odd, then $\gamma_{a^{\prime}, b^{\prime}}^{2}\left(\left(\begin{array}{c}{[n]} \\ \left.\left\lfloor a^{\prime}+b^{\prime}\right) / 2\right\rfloor\end{array}\right)\right)=0$. But we know by part (a)

$$
\gamma_{a^{\prime}, b^{\prime}}^{2}(\mathcal{F}) w\left(a^{\prime}, b^{\prime}\right) \leq\left(\begin{array}{c}
b^{\prime}-a^{\prime}-1 \\
\left(b^{\prime}-a^{\prime}-1\right) / 2-1
\end{array}\right)\left(\begin{array}{l}
n \\
b^{\prime}
\end{array}\right)\left(\begin{array}{l}
b^{\prime} \\
a^{\prime}
\end{array}\right) w\left(a^{\prime}, b^{\prime}\right) .
$$

Similarly to the previous cases, if $b^{\prime}-a^{\prime}$ is fixed, then the maximum of the right hand side is taken for some $a^{*}, b^{*}$ with $b^{*}-a^{*}=b^{\prime}-a^{\prime}$ and $a^{*}+b^{*}=n$ if $n$ is odd, and $a^{*}+b^{*}=n-1$ or $a^{*}+b^{*}=n+1$ if $n$ is even. Thus we can assume $\lfloor n / 2\rfloor=\left(a^{*}+b^{*}-1\right) / 2$. On the other hand, since $b^{*}-a^{*}$ is odd, we have

$$
3 \gamma_{0, n}^{3}\left(\left(\begin{array}{c}
{[n]} \\
\left(a^{*}+b^{*}-1\right) / 2
\end{array}\right)\right) \geq\left(\begin{array}{c}
n \\
b^{*}-1
\end{array}\right)\left(\begin{array}{c}
b^{*}-1 \\
a^{*}
\end{array}\right) \frac{1}{2}\left(\begin{array}{c}
b^{*}-a^{*}-1 \\
\left(b^{*}-a^{*}-1\right) / 2
\end{array}\right)\left(\begin{array}{c}
b^{*}-1-a^{*} \\
\frac{a^{*}+b^{*}-1}{2}-n+b^{*}-1
\end{array}\right),
$$

by counting only those triples where two of the sets have intersection of size $a^{*}$ and union of size $b^{*}-1$. We can pick first the $\left(b^{*}-1\right)$-set $B$ and the $a^{*}$-set $A$ in $\left(\begin{array}{c}n \\ b^{*}-1\end{array}\right)\left(\begin{array}{c}b^{*}-1 \\ a^{*}\end{array}\right)$ ways. Then among $\left\{G \in\left(\begin{array}{c}{[n]} \\ \left\lfloor\left(a^{*}+b^{*}\right) / 2\right\rfloor\end{array}\right): A \subset G \subset B\right\}$ we can pick a pair $G_{1}, G_{2}$ with $G_{1} \cap G_{2}=A$, $G_{1} \cup G_{2}=B$ in $\left(\begin{array}{c}b^{*}-a^{*}-1 \\ \left(b^{*}-a^{*}-1\right) / 2\end{array}\right) / 2$ ways and then the third set contains the complement of $B$ and does not intersect $A$. Using that $\left(\begin{array}{c}b^{*}-a^{*}-1 \\ \frac{a^{*}+b^{*}-1}{2}-n+b^{*}-1\end{array}\right) \geq\left(\begin{array}{c}b^{*}-a^{*}-1 \\ \left(b^{*}-a^{*}-1\right) / 2-1\end{array}\right)$, this implies

$$
3 \gamma_{0, n}^{3}(\mathcal{F}) \leq 3 n^{2} \gamma_{0, n}^{3}\left(\left(\begin{array}{c}
{[n]} \\
\left(a^{*}+b^{*}-1\right) / 2
\end{array}\right)\right) \frac{n-b^{*}+1}{\left(b^{*}-a^{*}-1\right) / 2} \leq 3 n^{3} \gamma_{0, n}^{3}\left(\left(\begin{array}{c}
{[n]} \\
\lfloor n / 2\rfloor
\end{array}\right)\right),
$$

as $b^{*} \geq n / 2$. 
Theorem 4.2 (a) For any antichain $\mathcal{F} \subseteq 2^{[n]}$ we have $\beta_{i}^{2}(\mathcal{F}) \leq \beta_{i}^{2}\left(\left(\begin{array}{c}{[n]} \\ j(i)\end{array}\right)\right)$, where $j(i)=$ $i+\lfloor(n-i) / 3\rfloor$ if $n-i \equiv 0,1 \bmod 3$ and $j(i)=i+\lceil(n-i) / 3\rceil$ if $n-i \equiv 2 \bmod 3$.

(b) For every $r \geq 3$ and $i \leq n$ there exists $j(r, i, n)$ such that $\beta_{i}^{r}(\mathcal{F}) \leq n^{2 r+1} \beta_{i}^{r}\left(\left(\begin{array}{c}{[n]} \\ j(r, i, n)\end{array}\right)\right)$ holds for any antichain $\mathcal{F} \subseteq 2^{[n]}$.

Proof First we prove (a) for the special case $i=0$. Let $\mathcal{F} \subseteq 2^{[n]}$ be an antichain, and let $\overline{\mathcal{F}}=\{\bar{F}: F \in \mathcal{F}\}$, where $\bar{F}=[n] \backslash F$. As $\mathcal{F}$ is an antichain, so is $\overline{\mathcal{F}}$, and thus $\mathcal{F} \cup \overline{\mathcal{F}}$ is $P_{3}$-free. Note that for every pair $F_{1}, F_{2} \in \mathcal{F}$ with $\left|F_{1} \cap F_{2}\right|=0$ and $\left|F_{1} \cup F_{2}\right|<[n]$, we have two 2-chains $F_{1} \subsetneq \overline{F_{2}}$ and $F_{2} \subsetneq \overline{F_{1}}$. Also, every 2-chain in $\mathcal{F} \cup \overline{\mathcal{F}}$ comes from a pair $F_{1}, F_{2} \in \mathcal{F}$ with $\left|F_{1} \cap F_{2}\right|=0$ and $F_{1} \cup F_{2} \neq[n]$.

Let us take the canonical partition of $\mathcal{F} \cup \overline{\mathcal{F}}$ into $\mathcal{F}_{1} \cup \mathcal{F}_{2}$ and introduce the weight function $w(F)=\frac{1}{2}\left(_{\lceil|F| / 2\rceil}^{|F|}\right)$ if $F \in \mathcal{F}_{2}, \bar{F} \notin \mathcal{F}_{2}$ and $w(F)=1 / 2$ if $F \in \mathcal{F}_{2}, \bar{F} \in \mathcal{F}_{2}$. By the above, the number of disjoint pairs in $\mathcal{F}$ is at most $\sum_{F \in \mathcal{F}_{2}} w(F)$. Observe that this weight function does not depend only on the size of $F$. However, the modified weight function $w^{\prime}(F):=\frac{1}{2}\left(\begin{array}{c}|F| \\ \lceil|F| / 2\rceil\end{array}\right)$ depends only on the size of $F$ and obviously $w(F) \leq w^{\prime}(F)$ holds for all $F$ 's. Thus we can apply a theorem of Katona (mentioned in the introduction, Theorem 3 in [19]) for $w^{\prime}$ to obtain that $\sum_{F \in \mathcal{F}_{2}} w^{\prime}(F)$ is maximized when $\mathcal{F}_{2}=\left(\begin{array}{c}{[n]} \\ \lceil 2 n / 3\rceil\end{array}\right)$. As $\mathcal{F}_{2}$ does not contain complement pairs, this family also maximizes $w$.

To see the general statement of (a), we can apply the special case to any $I \subseteq[n]$ and $\mathcal{F}_{I}=\{F \backslash I: I \subseteq F \in \mathcal{F}\}$. We obtain

$$
\beta_{i}^{2}(\mathcal{F})=\sum_{I \in\left(\begin{array}{c}
{[n]} \\
i
\end{array}\right)} \beta_{0}^{2}\left(\mathcal{F}_{I}\right) \leq\left(\begin{array}{c}
n \\
i
\end{array}\right) \beta_{0}^{2}\left(\left(\begin{array}{c}
{[n-i]} \\
j(i)-i
\end{array}\right)\right)=\beta_{i}^{2}\left(\left(\begin{array}{c}
{[n]} \\
j(i)
\end{array}\right)\right) .
$$

To see (b), let $\mathcal{F} \subseteq 2^{[n]}$ be an antichain. The next chain of inequalities finishes the proof. The penultimate inequality follows from Theorem 4.1 .

$$
\begin{aligned}
\beta_{i}^{r}(\mathcal{F})=\sum_{j=i+1}^{n} \gamma_{i, j}^{r}(\mathcal{F}) & \leq n \max _{j: i+1 \leq j \leq n} \gamma_{i, j}^{r}(\mathcal{F}) \\
& \leq n^{2 r+1} \max _{j: i+1 \leq j \leq n} \gamma_{i, j}^{r}\left(\left(\begin{array}{c}
{[n]} \\
\lfloor(i+j) / 2\rfloor
\end{array}\right)\right) \leq n^{2 r+1} \beta_{i}^{r}\left(\left(\begin{array}{c}
{[n]} \\
j(r, i, n)
\end{array}\right)\right),
\end{aligned}
$$

where $j(r, i, n)=\left\lfloor\left(i+j^{*}\right) / 2\right\rfloor$ with $j^{*}$ being the value of $j$ that maximizes $\gamma_{i, j}^{r}\left(\left(\begin{array}{c}{[n]} \\ \lfloor(i+j) / 2\rfloor\end{array}\right)\right)$.

Proof of Theorem 1.9 Let $Q_{1}, Q_{2}$ be non-empty posets and let us consider the canonical partition of a $P_{l\left(Q_{1} \otimes_{r} Q_{2}\right)+1}$-free family $\mathcal{F} \subseteq 2^{[n]}$. Then in any copy of $Q_{1} \otimes_{r} Q_{2}$ in $\mathcal{F}$, if $F_{1}, \ldots, F_{r}$ correspond to the $r$ middle elements forming an antichain, we must have $F_{1}, \ldots, F_{r} \in \mathcal{F}_{l\left(Q_{1}\right)+1}$. Also, if a copy of $Q_{1} \otimes_{r} Q_{2}$ contains $F_{1}, \ldots, F_{r}$, then the sets corresponding to the $Q_{1}$ part of $Q_{1} \otimes_{r} Q_{2}$ must be contained in $\bigcap_{l=1}^{r} F_{l}$, while the sets corresponding to the $Q_{2}$ part of $Q_{1} \otimes_{r} Q_{2}$ must contain $\bigcup_{l=1}^{r} F_{l}$. Therefore the number of copies of $Q_{1} \otimes_{r} Q_{2}$ in $\mathcal{F}$ that contain $F_{1}, \ldots, F_{r}$ is at most $L a\left(\left|\bigcap_{l=1}^{r} F_{l}\right|, P_{l\left(Q_{1}\right)+1}, Q_{1}\right)$. $L a\left(n-\left|\bigcup_{i=1}^{r} F_{l}\right|, P_{l\left(Q_{2}\right)+1}, Q_{2}\right)$. We obtained that the total number of copies of $Q_{1} \otimes_{r} Q_{2}$ in $\mathcal{F}$ is at most

$$
\sum_{F_{1}, \ldots, F_{r} \in \mathcal{F}_{l\left(Q_{1}\right)+1}} L a\left(\left|\bigcap_{l=1}^{r} F_{l}\right|, P_{l\left(Q_{1}\right)+1}, Q_{1}\right) \cdot \operatorname{La}\left(n-\left|\bigcup_{l=1}^{r} F_{l}\right|, P_{l\left(Q_{2}\right)+1}, Q_{2}\right) .
$$

If $r \geq 2$, then grouping the summands in (1) according to the pair $\left(\left|\bigcap_{l=1}^{r} F_{l}\right|,\left|\bigcup_{l=1}^{r} F_{l}\right|\right)$ we obtain

$$
L a\left(n, P_{l\left(Q_{1} \otimes_{r} Q_{2}\right)+1}, Q_{1} \otimes_{r} Q_{2}\right) \leq \gamma^{r}\left(\mathcal{F}_{l\left(Q_{1}\right)+1}\right) \cdot \mathbf{w},
$$


where the $(i, j)$ th coordinate of $\mathbf{w}$ is $L a\left(i, P_{l\left(Q_{1}\right)}, Q_{1}\right) \cdot L a\left(n-j, P_{l\left(Q_{2}\right)+1}, Q_{2}\right)$. Clearly, we have

$$
\gamma^{r}\left(\mathcal{F}_{l\left(Q_{1}\right)+1}\right) \cdot \mathbf{w} \leq n^{2} \max _{i, j} \gamma_{i, j}^{r}\left(\mathcal{F}_{l\left(Q_{1}\right)+1}\right) w(i, j) \leq n^{2 r+2} \max _{i, j} \gamma_{i, j}^{r}\left(\left(\begin{array}{c}
{[n]} \\
\lfloor(i+j) / 2\rfloor
\end{array}\right)\right) w(i, j),
$$

where the last inequality follows from Theorem 4.1. We can calculate $\gamma_{i, j}^{r}\left(\left(_{\lfloor(i+j) / 2\rfloor}^{[n]}\right)\right)$ by picking the union of size $j$ and the intersection of size $i$ first, which finishes the proof of the upper bound of part (a).

To see the furthermore part, suppose that the above maximum is obtained when $i$ takes the value $i^{*}$ and $j$ takes the value $j^{*}$. We know that there exist two families $\mathcal{F}_{1, i^{*}} \subseteq 2^{\left[i^{*}\right]}$ and $\mathcal{F}_{2, n-j^{*}} \subseteq 2^{\left[n-j^{*}\right]}$, both unions of full levels, integers $k_{1}, k_{2}$ and constants $C_{1}, C_{2}$ such that $C_{1}\left(i^{*}\right)^{k_{1}} c\left(Q_{1}, \mathcal{F}_{1, i^{*}}\right) \geq L a\left(i^{*}, P_{l\left(Q_{1}\right)+1}, Q_{1}\right)$ and $C_{2}\left(j^{*}\right)^{k_{2}} c\left(Q_{2}, \mathcal{F}_{2, n-j^{*}}\right) \geq$ $L a\left(n-j^{*}, P_{l\left(Q_{2}\right)+1}, Q_{2}\right)$ hold. Therefore, by the upper bound already proven, we know that $\operatorname{La}\left(n, P_{l\left(Q_{1} \otimes_{r} Q_{2}\right)+1}, Q_{1} \otimes_{r} Q_{2}\right)$ is at most

$$
n^{2 r+2} C_{1}\left(i^{*}\right)^{k_{1}} C_{2}\left(j^{*}\right)^{k_{2}} \gamma_{i^{*} j^{*}}^{r}\left(\left(\begin{array}{c}
n \\
\left\lfloor\left(i^{*}+j^{*}\right) / 2\right\rfloor
\end{array}\right)\right) L a\left(i^{*}, P_{l\left(Q_{1}\right)+1}, Q_{1}\right) L a\left(n-i^{*}, P_{l\left(Q_{2}\right)+1}, Q_{2}\right) .
$$

If $\mathcal{F}_{1, i^{*}}$ consists of levels of set sizes $h_{1}, \ldots, h_{l\left(Q_{1}\right)}$ and $\mathcal{F}_{2, n-i^{*}}$ consists of levels of set sizes $h_{1}^{\prime}, \ldots, h_{l\left(Q_{2}\right)}^{\prime}$, then for the family

$$
\mathcal{F}:=\left(\begin{array}{c}
{[n]} \\
h_{1}
\end{array}\right) \cup \ldots\left(\begin{array}{c}
{[n]} \\
h_{l\left(Q_{1}\right)}
\end{array}\right) \cup\left(\begin{array}{c}
{[n]} \\
\left\lfloor\left(i^{*}+j^{*}\right) / 2\right\rfloor
\end{array}\right) \cup\left(\begin{array}{c}
{[n]} \\
j^{*}+h_{1}^{\prime}
\end{array}\right) \cup \cdots \cup\left(\begin{array}{c}
{[n]} \\
j^{*}+h_{l\left(Q_{2}\right)}^{\prime}
\end{array}\right),
$$

we have $c\left(Q_{1} \otimes Q_{2}, \mathcal{F}\right) \geq \gamma_{i^{*} j^{*}}^{r}\left(\left(\begin{array}{c}n \\ \left\lfloor\left(i^{*}+j^{*}\right) / 2\right\rfloor\end{array}\right)\right) L a\left(i^{*}, P_{l\left(Q_{1}\right)+1}, Q_{1}\right) L a(n-$ $\left.i^{*}, P_{l\left(Q_{2}\right)+1}, Q_{2}\right)$. Therefore, with $k=2 r+2+k_{1}+k_{2}$ the family $\mathcal{F}$ shows that Conjecture 1.5 almost holds for the pair $P_{l\left(Q_{1} \otimes_{r} Q_{2}\right)+1}, Q_{1} \otimes_{r} Q_{2}$.

If $r=1$, then $\cup F_{1}=\cap F_{1}=F_{1}$, so (1) becomes

$$
\sum_{F \in \mathcal{F}_{l\left(Q_{1}\right)+1}} L a\left(|F|, P_{l\left(Q_{1}\right)+1}, Q_{1}\right) \cdot L a\left(n-|F|, P_{l\left(Q_{2}\right)+1}, Q_{2}\right) .
$$

We can apply Corollary 3.2 with $l=k=1$ and $w(i)=\operatorname{La}\left(i, P_{l\left(Q_{1}\right)+1}, Q_{1}\right)$. $L a\left(i, P_{l\left(Q_{2}\right)+1}, Q_{2}\right)$ to obtain

$L a\left(n, P_{l\left(Q_{1} \otimes Q_{2}\right)+1}, Q_{1} \otimes Q_{2}\right) \leq \max _{0 \leq i \leq n}\left\{\left(\begin{array}{c}n \\ i\end{array}\right) L a\left(i, P_{l\left(Q_{1}\right)+1}, Q_{1}\right) L a\left(n-i, P_{l\left(Q_{2}\right)+1}, Q_{2}\right)\right\}$ as required.

As the proofs are almost identical, we only show the "strongly holds" case of the furthermore part of (b). Suppose that the above maximum is obtained when $i$ takes the value $i^{*}$. We know that there exist two families $\mathcal{F}_{1, i^{*}} \subseteq 2^{\left[i^{*}\right]}$ and $\mathcal{F}_{2, n-i^{*}} \subseteq 2^{\left[n-i^{*}\right]}$, both unions of full levels, such that $c\left(Q_{1}, \mathcal{F}_{1, i^{*}}\right)=\operatorname{La}\left(i^{*}, P_{l\left(Q_{1}\right)+1}, Q_{1}\right)$ and $c\left(Q_{2}, \mathcal{F}_{2, n-i^{*}}\right)=$ $L a\left(n-i^{*}, P_{l\left(Q_{2}\right)+1}, Q_{2}\right)$ hold. If $\mathcal{F}_{1, i^{*}}$ consists of levels of set sizes $j_{1}, \ldots, j_{l\left(Q_{1}\right)}$ and $\mathcal{F}_{2, n-i^{*}}$ consists of levels of set sizes $j_{1}^{\prime}, \ldots, j_{l\left(Q_{2}^{\prime}\right)}^{\prime}$, then for the family

$$
\mathcal{F}:=\left(\begin{array}{c}
{[n]} \\
j_{1}
\end{array}\right) \cup \ldots\left(\begin{array}{c}
{[n]} \\
j_{l\left(Q_{1}\right)}
\end{array}\right) \cup\left(\begin{array}{c}
{[n]} \\
i^{*}
\end{array}\right) \cup\left(\begin{array}{c}
{[n]} \\
i^{*}+j_{1}^{\prime}
\end{array}\right) \cup \cdots \cup\left(\begin{array}{c}
{[n]} \\
i^{*}+j_{l\left(Q_{2}\right)}^{\prime}
\end{array}\right)
$$

we have $c\left(Q_{1} \otimes Q_{2}, \mathcal{F}\right)=\left(\begin{array}{c}n \\ i^{*}\end{array}\right) L a\left(i^{*}, P_{l\left(Q_{1}\right)+1}, Q_{1}\right) L a\left(n-i^{*}, P_{l\left(Q_{2}\right)+1}, Q_{2}\right)$.

Proof of Theorem 1.10 The proof goes very similarly to the proof of Theorem 1.9. Let us consider the canonical partition of a $P_{l(Q \otimes r)+1}$-free family $\mathcal{F} \subseteq 2^{[n]}$. Then in any copy 
of $Q \otimes r$ in $\mathcal{F}$, if $F_{1}, \ldots, F_{r}$ correspond to the $r$ top elements forming an antichain, we must have $F_{1}, \ldots, F_{r} \in \mathcal{F}_{l(Q)+1}$. Also, if a copy of $Q \otimes r$ contains $F_{1}, \ldots, F_{r}$, then the sets corresponding to the other elements of the poset must be contained in $\bigcap_{l=1}^{r} F_{l}$. Let $j=\left|\bigcap_{l=1}^{r} F_{l}\right|$. Then the number of copies of $Q \otimes r$ in $\mathcal{F}$ that contain $F_{1}, \ldots, F_{r}$ is at most $L a\left(j, P_{l(Q)+1}, Q\right)$. If $r \geq 2$, we obtain

$$
c(Q \oplus r, \mathcal{F}) \leq \beta^{r}\left(\mathcal{F}_{l(Q)+1}\right) \cdot \mathbf{w},
$$

where the $j$ th coordinate of $\mathbf{w}$ is $L a\left(j, P_{l(Q)+1}, Q\right)$. Clearly, we have

$$
\beta^{r}\left(\mathcal{F}_{l(Q)+1}\right) \cdot \mathbf{w} \leq n \max _{i} \beta_{i}^{r}\left(\mathcal{F}_{l(Q)+1}\right) w(i) \leq n^{2 r+2} \max _{i} \beta_{i}^{r}\left(\left(\begin{array}{c}
{[n]} \\
j(r, i, n)
\end{array}\right)\right) w(i),
$$

where the last inequality follows from Theorem 4.2. We have $\beta_{i}^{r}\left(\left(\begin{array}{c}{[n]} \\ j(r, i, n)\end{array}\right)\right)=$ $\left(\begin{array}{c}n \\ i\end{array}\right) \beta_{0}^{r}\left(\left(\begin{array}{c}{[n-i]} \\ j(r, i, n)-i\end{array}\right)\right)$ by picking the intersection of size $i$ first.

To see the furthermore part of (a), let $i^{*}$ be the value of $i$ for which the above maximum is attained. Assume Conjecture 1.5 almost holds for the pair $P_{l(Q)+1}, Q$. Then there exists a family $\mathcal{F}_{i^{*}}=\left(\begin{array}{c}{\left[i^{*}\right]} \\ h_{1}\end{array}\right) \cup \cdots \cup\left(\begin{array}{c}{\left[i^{*}\right]} \\ h_{l(Q)}\end{array}\right)$ with $L a\left(i^{*}, P_{l(Q)+1}, Q\right) \leq C i^{*{ }^{k}} c\left(Q, \mathcal{F}_{i^{*}}\right) \leq$ $C n^{k} c\left(Q, \mathcal{F}_{i^{*}}\right)$ for some constants $C$ and $k$. Then for the family $\mathcal{F}^{*}=\left(\begin{array}{c}{[n]} \\ h_{1}\end{array}\right) \cup \cdots \cup\left(\begin{array}{c}{[n]} \\ h_{l(Q)}\end{array}\right) \cup$ $\left(\begin{array}{c}{[n]} \\ j\left(r, i^{*}, n\right)\end{array}\right)$, we have

$c\left(Q \oplus r, \mathcal{F}^{*}\right) \geq \beta_{i^{*}}^{r}\left(\left(\begin{array}{c}{[n]} \\ j\left(r, i^{*}, n\right)\end{array}\right)\right) c\left(Q, \mathcal{F}_{i^{*}}\right) \geq\left(\begin{array}{c}n \\ i^{*}\end{array}\right) \beta_{0}^{r}\left(\left(\begin{array}{c}{\left[n-i^{*}\right]} \\ j\left(r, i^{*}, n\right)-i^{*}\end{array}\right)\right) \frac{1}{C n^{k}} \operatorname{La}\left(n, i^{*}, Q\right)$,

therefore $\mathcal{F}^{*}$ with $C^{\prime}=C$ and $k^{\prime}=2 r+2+k$ shows that Conjecture 1.5 almost holds for the pair $P_{l(Q)+2}, Q \oplus r$.

If $r=1$, then $\left|\cap F_{1}\right|=\left|F_{1}\right|$, so applying Corollary 3.2 with $l=k=1$ we obtain

$$
c(Q \oplus 1, \mathcal{F}) \leq \sum_{F \in \mathcal{F}_{l(Q)+1}} L a\left(|F|, P_{l(Q)+1}, Q\right) \leq \max _{0 \leq i \leq n}\left\{\left(\begin{array}{l}
n \\
i
\end{array}\right) L a\left(i, P_{l(Q)+1}, Q\right)\right\} .
$$

The proof of the furthermore part of (b) is analogous to the previous ones and is left to the reader.

Proof of Corollary 1.11 We proceed by induction on the number of levels. The base case is guaranteed by Sperner's Theorem 1.1. The inductive step follows by applying Theorem 1.10 as $K_{r_{1}, \ldots, r_{l}}=K_{r_{1}, \ldots, r_{l-1}} \oplus r_{l}$.

Proof of Corollary 1.12 We proceed by induction on the number of levels. The base case is guaranteed by Sperner's Theorem 1.1. Suppose the statement has been proved for all complete multipartite posets satisfying the condition with height smaller than $l$ and consider $K_{r_{1}, r_{2}, \ldots, r_{l}}$. We know that there exists an $i$ with $1 \leq i \leq l$ such that $r_{i}=1$. If $1<i<l$, then the inductive step will follow by applying the furthermore part of Theorem 1.9 to $Q_{1}=K_{r_{1}, \ldots, r_{i-1}}$ and $Q_{2}=K_{r_{i+1}, \ldots, r_{l}}$. If $i=l$, then the inductive step will follow by applying the furthermore part of Theorem 1.10 to $Q=K_{r_{1}, \ldots, r_{l-1}}$ and $r=1$. The case $i=1$ follows from $c\left(K_{r_{1}, \ldots, r_{l}}, \mathcal{F}\right)=c\left(K_{r_{l}, \ldots, r_{1}}, \overline{\mathcal{F}}\right)$, where $\overline{\mathcal{F}}=\{[n] \backslash F: F \in \mathcal{F}\}$.

Proof of Theorem 1.13 We only give the sketch of the proof as it is very similar to previous ones. Consider a $P_{l+3}$-free family $\mathcal{F} \subseteq 2^{[n]}$ and its canonical partition. If $l=1$, then we count the number of copies of $K_{r, 1, s}$ according to the set $F \in \mathcal{F}_{2}$ that plays the role 
Fig. 3 The Hasse diagrams of the posets $B^{+}$and $B^{++}$

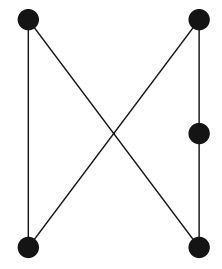

$B^{+}$

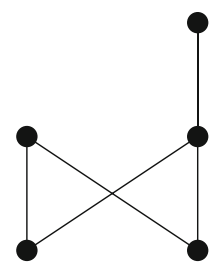

$B^{++}$

of the middle element of $K_{r, 1, s}$. The number of copies that contain $F$ is not more than $\left(\underset{r}{\left(\begin{array}{c}|F| \\ \lfloor\mid / 2\rfloor\end{array}\right)}\right)\left(\underset{S}{\left(\begin{array}{c}n-|F| \\ \lfloor(n-|F|) / 2\rfloor\end{array}\right)}\right)$. Applying Corollary 3.2 with $l=k=1$ and $w(i)=\left(\begin{array}{c}i \\ \lfloor i / 2\rfloor \\ r\end{array}\right)\left(\begin{array}{c}\left(\begin{array}{c}n-i \\ \lfloor(n-i) / 2\rfloor \\ s\end{array}\right)\end{array}\right)$ yields $c\left(K_{r, 1, s}, \mathcal{F}\right) \leq \max _{i}\left(\begin{array}{c}n \\ i\end{array}\right)\left(\begin{array}{c}\left(\begin{array}{c}i \\ \lfloor i / 2\rfloor \\ r\end{array}\right) \\ r\end{array}\right)\left(\begin{array}{c}\left(\begin{array}{c}n-i \\ (n-i) / 2\rfloor\end{array}\right) \\ s\end{array}\right)$. Let $i^{*}$ be the value of $i$ for which this maximum is attained. Then the family $\left(\begin{array}{c}{[n]} \\ \left\lfloor i^{*} / 2\right\rfloor\end{array}\right) \cup\left(\begin{array}{c}{[n]} \\ i^{*}\end{array}\right) \cup\left(\begin{array}{c}{[n]} \\ \left\lfloor\left(n+i^{*}\right) / 2\right\rfloor\end{array}\right)$ contains exactly that many copies of $K_{r, 1, s}$.

If $l \geq 2$, then we count the number of copies of $K_{r, 1,1, \ldots, 1, s}$ according to the sets $F_{2} \in \mathcal{F}_{2}$ and $F_{l+1} \in \mathcal{F}_{l+1}$ playing the role of the elements on the second and $(l+1)$ st level of $K_{r, 1,1, \ldots, 1, s}$. For a fixed pair $F_{2} \in \mathcal{F}_{2}$ and $F_{l+1} \in \mathcal{F}_{l+1}$ with $F_{2} \subset F_{l+1}$, the number of copies of $K_{r, 1,1, \ldots, 1, s}$ containing $F_{2}$ and $F_{l+1}$ is at most $\left(\begin{array}{c}\left(\begin{array}{c}\left|F_{2}\right| \\ \left\lfloor F_{2} \mid / 2\right\rfloor\end{array}\right) \\ P^{\prime}\end{array}\right)\left(\begin{array}{c}\left(\begin{array}{c}n-\left|F_{l+1}\right| \\ \left.\left\lfloor n-\left|F_{l+1}\right|\right) / 2\right\rfloor\end{array}\right) \\ )\end{array}\right) L a\left(\left|F_{l+1}\right|-\right.$ $\left.\left|F_{2}\right|, P_{l-1}, P_{l-2}\right)$. The value of $L a\left(\left|F_{l+1}\right|-\left|F_{2}\right|, P_{l-1}, P_{l-2}^{r}\right)$ is given by Theorem 1.3. So we can apply Corollary 3.2 with $l=k=2$ and $w(i, j)=\left(\begin{array}{c}\left(\begin{array}{c}i \\ i / 2\rfloor\end{array}\right) \\ r\end{array}\right)\left(\begin{array}{c}\left(\begin{array}{c}n-j \\ \lfloor n-j) / 2\rfloor \\ s\end{array}\right) \\ \text { s }\end{array}\right) \operatorname{La}(j-$ $\left.i, P_{l-1}, P_{l-2}\right)$ to obtain $c\left(K_{r, 1,1 \ldots, 1, s}, \mathcal{F}\right) \leq \max _{i, j}\left(\begin{array}{c}n \\ j\end{array}\right)\left(\begin{array}{c}j \\ i\end{array}\right)\left(\underset{r}{\left(\begin{array}{c}i \\ \lfloor i / 2\rfloor\end{array}\right)}\right)\left(\begin{array}{c}\left(\begin{array}{c}n-j \\ \lfloor(n-j) / 2\rfloor \\ s\end{array}\right) \\ r\end{array}\right) L a(j-$ $\left.i, P_{l-1}, P_{l-2}\right)$. Let $i^{*}$ and $j^{*}$ be the values of $i$ and $j$ for which this maximum is attained. Then the family consisting of $\left(\begin{array}{c}{[n]} \\ \left\lfloor i^{*} / 2\right\rfloor\end{array}\right),\left(\begin{array}{c}{[n]} \\ i^{*}\end{array}\right),\left(\begin{array}{c}{[n]} \\ j^{*}\end{array}\right),\left(\begin{array}{c}{[n]} \\ \left\lfloor\left(n+j^{*}\right) / 2\right\rfloor\end{array}\right)$ and the $l-2$ full levels determined by Theorem 1.3 contains exactly that many copies of $K_{r, 1,1, \ldots, 1, s}$.

There are several other complete multi-partite posets for which one can determine the levels that form an almost optimal family. For example, using Theorem 4.2 (a), one can prove that $L a\left(n, P_{3}, K_{p, 2}\right) \leq n c\left(K_{p, 2}, \mathcal{F}\right)$ where $\mathcal{F}=\left(\begin{array}{c}{[n]} \\ i\end{array}\right) \cup\left(\begin{array}{c}{[n]} \\ j\end{array}\right)$ with $i=\left(\frac{2^{p-1}}{3+2^{p}}+o(1)\right) n$ and $j=\left(\frac{1+2^{p}}{3+2^{p}}+o(1)\right) n$. In particular $L a\left(n, P_{3}, K_{p, 2}\right)=2^{\left(c_{p}+o(1)\right) n}$, where $c_{p}=\frac{2+p \cdot 2^{p}}{3+2^{p}}+$ $h\left(\frac{2^{p}}{3+2^{p}}\right)+\frac{3}{3+2^{p}} h(2 / 3)$. Recall that $h$ denotes the binary entropy function.

Let us finish this section by some remarks about $K_{2,2}=B$, as there exist several extremal results concerning $B$. Let us consider the following two posets that contain $B$ : $B^{+}$and $B^{++}$have five elements $a, b, c, d, e$ such that $a<B^{+} c, e$ and $b<B^{+} c, d$ and also $d<_{B^{+}} e$, while $a, b<_{B^{++}} c, d$ and $d<_{B^{++}} e$ (Fig. 3). By results of DeBonis, Katona, and Swanepoel [5] and Methuku and Tompkins [23] we know that $\operatorname{La}\left(n, B, P_{1}\right)=$ $L a\left(n, B^{+}, P_{1}\right)=\left(\begin{array}{c}n \\ \lfloor n / 2\rfloor\end{array}\right)+\left(\begin{array}{c}n \\ \lfloor n / 2\rfloor+1\end{array}\right)$, and as $B^{++}$contains a copy of $B^{+}$we have $L a\left(n, B^{+}, P_{1}\right) \leq \operatorname{La}\left(n, B^{++}, P_{1}\right)$. It is natural to ask how many copies of $B$ can a $B^{+}$-free or $B^{++}$-free family in $2^{[n]}$ contain, especially that the largest $B^{+}$-free family does not contain any. Obviously, a $P_{3}$-free poset is both $B^{+}$-free and $B^{++}$-free, therefore we obtain the inequality $L a\left(n, P_{3}, B\right) \leq L a\left(n, B^{+}, B\right) \leq L a\left(n, B^{++}, B\right)$. The next proposition shows that these functions are asymptotically equal.

\section{Proposition 4.3}

$$
L a\left(n, P_{3}, B\right) \leq L a\left(n, B^{+}, B\right) \leq L a\left(n, B^{++}, B\right)=(1+o(1)) L a\left(n, P_{3}, B\right) .
$$


Note that $L a\left(n, P_{3}, B\right)=2^{\left(c_{2}+o(1)\right) n}$, where $c_{2}=10 / 7+h(4 / 7)+3 h(2 / 3) / 7$ by the remarks made after the proof of Theorem 1.13.

Proof The first two inequalities are true by definition. Let $\mathcal{F} \subseteq 2^{[n]}$ be $B^{++}$-free, and consider its canonical partition (note that $P_{5}$ contains a copy of $B^{++}$, thus $\mathcal{F}$ is $P_{5}$-free).

Consider first the family $\mathcal{S} \subseteq \mathcal{F}_{2}$ of sets that appear in 4-chains in $\mathcal{F}$ (they must be the second smallest in those chains). Note that if $S \in \mathcal{S}$ with $F_{1} \subsetneq S \subsetneq F_{3} \subsetneq F_{4}$, then $S$ is not comparable to any other set $F$ of $\mathcal{F}$ as $F, F_{1}, S, F_{3}, F_{4}$ would form a copy of $B^{++}$both if $S \subsetneq F$ or $F \subsetneq S$. Therefore every set $S \in \mathcal{S}$ is contained in at most one copy of $B$ in $\mathcal{F}$. As $\mathcal{S} \subseteq \mathcal{F}_{2}$ is an antichain, we obtain $c(B, \mathcal{F})-c(B, \mathcal{F} \backslash \mathcal{S}) \leq\left(\begin{array}{c}n \\ \lfloor n / 2\rfloor\end{array}\right)$.

Clearly, $\mathcal{F}^{\prime}=\mathcal{F} \backslash \mathcal{S}$ is $P_{4}$-free. Let us consider its canonical partition and denote the resulting antichains by $\mathcal{F}_{1}^{\prime}, \mathcal{F}_{2}^{\prime}, \mathcal{F}_{3}^{\prime}$. Let $\mathcal{S}^{\prime} \subseteq \mathcal{F}_{2}^{\prime}$ be the family of middle sets of all 3-chains in $\mathcal{F}^{\prime}$. We know that for any $S \in \mathcal{S}^{\prime}$ there exist $F_{1}^{\prime}, F_{3}^{\prime} \in \mathcal{F}^{\prime}$ with $F_{1}^{\prime} \subsetneq S \subsetneq F_{3}^{\prime}$. Also, there cannot exist $F_{1}^{\prime \prime}, F_{3}^{\prime \prime}$ with $F_{1}^{\prime \prime} \subsetneq S \subsetneq F_{3}^{\prime \prime}$ as then $F_{1}^{\prime}, F_{1}^{\prime \prime}, S, F_{3}^{\prime}, F_{3}^{\prime \prime}$ would form a copy of $B^{++}$. So either there is a unique set $F^{\prime}$ that contains $S$ and potentially several sets that are contained in $S$ or there exists a unique $F^{\prime}$ contained in $S$ and several sets containing $S$. In the former case, if $S$ is contained in a copy of $B$, it can only be one of the top sets. Furthermore, if a copy of $B$ contains $S$, then it contains $F^{\prime}$ as otherwise this copy could be extended by $F^{\prime}$ to form a $B^{++}$. As the sets contained in $S$ form an antichain (they are a subfamily of $\left.\mathcal{F}_{1}^{\prime}\right)$, we obtain that the number of copies of $B$ containing $S$ is at most $\left(\left(\frac{|S|}{2}\right)_{2}\right)$. Similarly, if $S$ contains exactly one other set of $\mathcal{F}^{\prime}$, then the number of copies of $B$ containing $S$ is at $\operatorname{most}\left(\begin{array}{c}\left(\begin{array}{c}n-|S| \\ \left.\frac{n-|S|}{2}\right\rfloor\end{array}\right) \\ 2\end{array}\right)$. So introducing $\left.\left.w(i)=\max \left\{\left(\begin{array}{c}\left(\begin{array}{c}i \\ (i / 2\rfloor\end{array}\right) \\ 2\end{array}\right),\left(\begin{array}{c}(\lfloor n-i) / 2\rfloor \\ 2\end{array}\right)\right\}\right)\right\}$, we obtain that the total number of copies containing at least one element of $\mathcal{S}^{\prime}$ is at most $\sum_{S \in \mathcal{S}^{\prime}} w(|S|)$. By the special case $k=l=1$ of Corollary 3.2 we obtain that this expression is maximized over all antichains when $\mathcal{S}$ is a full level of $2^{[n]}$.

The weight function $w$ is symmetric, i.e. $w(i)=w(n-i)$ holds for any $i$, therefore it is enough to maximize $\left(\begin{array}{l}n \\ i\end{array}\right) w(i)$ over $n / 2 \leq i \leq n$. It is a routine exercise to see that $\left(\begin{array}{c}n \\ i\end{array}\right)\left(\begin{array}{c}\left(\begin{array}{c}i \\ \lfloor i / 2\rfloor\end{array}\right) \\ 2\end{array}\right)$ is maximized when $i=(4 / 5+o(1)) n$. Therefore the number of copies of $B$ that contain an element of $\mathcal{S}^{\prime}$ is at most $2^{h(4 / 5)+8 / 5+o(1)}$. We obtained that

$$
\begin{aligned}
& c(B, \mathcal{F}) \leq c\left(B, \mathcal{F} \backslash\left(\mathcal{S} \cup \mathcal{S}^{\prime}\right)\right)+\left(\begin{array}{c}
n \\
\lfloor n / 2\rfloor
\end{array}\right)+2^{h(4 / 5)+8 / 5+o(1)} \\
& \leq L a\left(n, P_{3}, B\right)+\left(\begin{array}{c}
n \\
\lfloor n / 2\rfloor
\end{array}\right)+2^{h(4 / 5)+8 / 5+o(1)} \\
& \quad=(1+o(1)) \operatorname{La}\left(n, P_{3}, B\right),
\end{aligned}
$$

as $h(4 / 5)+8 / 5=2.3219 \ldots<c_{2}$.

\section{Proof of Theorem 1.14}

Let us recall that Theorem 1.14 states that there does not exist $j$ with $\operatorname{La}\left(n, B, P_{2}\right)=$ $\Theta\left(L a\left(n, P_{j}, P_{2}\right)\right)$. By definition, we have $L a\left(n, P_{2}, P_{2}\right)=0$ and for any $j \geq 3$ we have $\operatorname{La}\left(n, P_{3}, P_{2}\right) \leq \operatorname{La}\left(n, P_{j}, P_{2}\right)$. Therefore it is enough to prove that

$$
\operatorname{La}\left(n, B, P_{2}\right)=o\left(\operatorname{La}\left(n, P_{3}, P_{2}\right)\right)
$$


holds. Let us remind the reader that by Theorem 1.3, we have $\operatorname{La}\left(n, P_{3}, P_{2}\right)=$ $\left(\begin{array}{c}n \\ \lceil 2 n / 3\rceil\end{array}\right)\left(\begin{array}{c}\lceil 2 n / 3\rceil \\ \lceil n / 3\rceil\end{array}\right)$. So the following theorem on $\operatorname{La}\left(n, B, P_{2}\right)$ will easily imply Theorem 1.14 .

Theorem $5.1 \frac{n}{2}\left(\begin{array}{c}n \\ \lfloor n / 2\rfloor\end{array}\right) \leq \operatorname{La}\left(n,\left\{B, P_{3}\right\}, P_{2}\right) \leq \operatorname{La}\left(n, B, P_{2}\right) \leq(2+o(1))\left(\begin{array}{c}n \\ \lceil n / 2\rceil\end{array}\right)\left(\begin{array}{l}{[n / 2\rceil} \\ \lceil n / 4\rceil\end{array}\right)$.

Proof The lower bound follows from the construction consisting of the two middle levels of $2^{[n]}$. To prove the upper bound, we will need several claims.

Claim 5.2 $\operatorname{La}\left(n, B, P_{2}\right)=(1+o(1)) \operatorname{La}\left(n,\left\{B, P_{3}\right\}, P_{2}\right)$.

Proof of Claim $\operatorname{La}\left(n, B, P_{2}\right) \geq \operatorname{La}\left(n,\left\{B, P_{3}\right\}, P_{2}\right)$ is true by definition. Let $\mathcal{F}$ be a $B$-free subfamily of $2^{[n]}$ and $\mathcal{M}=\left\{M \in \mathcal{F}: \exists F_{1}, F_{2} \in \mathcal{F} F_{1} \subsetneq M \subsetneq F_{2}\right\}$ be the family of middle sets. By the $B$-free property of $\mathcal{F}, \mathcal{M}$ is an antichain. As seen in the proof of Theorem 1.6 (c), for every $M \in \mathcal{M}$ there is exactly one $F_{1} \in \mathcal{F}$ and $F_{2} \in \mathcal{F}$ with $F_{1} \subsetneq$ $M \subsetneq F_{2}$. Therefore by Theorem 1.1 , we obtain $c\left(P_{2}, \mathcal{F}\right)-c\left(P_{2}, \mathcal{F} \backslash \mathcal{M}\right) \leq 2\left(\begin{array}{c}n \\ \lfloor n / 2\rfloor\end{array}\right)$. As $\mathcal{F} \backslash \mathcal{M}$ is $\left\{B, P_{3}\right\}$-free and $2\left(\begin{array}{c}n \\ \lfloor n / 2\rfloor\end{array}\right)=o\left(\operatorname{La}\left(n,\left\{B, P_{3}\right\}, P_{2}\right)\right)$, the claim follows.

Claim 5.3 Let $\mathcal{F} \subseteq 2^{[n]}$ be a $P_{3}$-free family and consider its canonical partition. Then the number of 2-chains in $\mathcal{F}$ that contain a set $F$ with either $|F|>\lfloor n / 2\rfloor$ and $F \in \mathcal{F}_{1}$ or $|F|<\lceil n / 2\rceil$ and $F \in \mathcal{F}_{2}$ is at most $\left(\begin{array}{c}n \\ \lfloor n / 2\rfloor\end{array}\right)\left(\begin{array}{c}{[n / 2\rceil} \\ \lceil n / 4\rceil\end{array}\right)$.

Proof of Claim Corollary 3.2 with $k=2$ and $w\left(F_{1}, F_{2}\right)=\frac{1}{\left(\begin{array}{c}n \\ \left|F_{2}\right|\end{array}\right)\left(\begin{array}{l}\left|F_{2}\right| \\ \left|F_{1}\right|\end{array}\right.}$ gives

$$
\sum_{\substack{F_{1} \subset F_{2}, F_{1}, F_{2} \in \mathcal{F}}} \frac{1}{\left(\begin{array}{c}
n \\
\left|F_{2}\right|
\end{array}\right)\left(\begin{array}{l}
\left|F_{2}\right| \\
\left|F_{1}\right|
\end{array}\right)} \leq 1 .
$$

Here a summand, in which $\left|F_{1}\right|>\lfloor n / 2\rfloor$ or $\left|F_{2}\right|<\lceil n / 2\rceil$ holds, has value at least $\frac{1}{\left(\begin{array}{c}n \\ \lfloor n / 2\rfloor\end{array}\right)\left(\begin{array}{l}{[n / 2]} \\ {[n / 4]}\end{array}\right]}$, thus the number of such summands is at most $\left(\begin{array}{c}n \\ \lfloor n / 2\rfloor\end{array}\right)\left(\begin{array}{l}{[n / 2\rceil} \\ \lceil n / 4\rceil\end{array}\right)$.

Claim 5.4 Let $\mathcal{F} \subseteq 2^{[n]}$ be a $\left\{P_{3}, B\right\}$-free family and consider its canonical partition. Suppose that $|F| \leq\lfloor n / 2\rfloor$ holds for all $F \in \mathcal{F}_{1}$ and $|F| \geq\lceil n / 2\rceil$ holds for all $F \in \mathcal{F}_{2}$. Then we have $c\left(P_{2}, \mathcal{F}\right) \leq\left(\begin{array}{c}n \\ \lceil n / 2\rceil\end{array}\right)\left(\begin{array}{c}\lceil n / 2\rceil \\ \lceil n / 4\rceil\end{array}\right)$.

Proof of Claim. Let us count the pairs $\left(\left\{F_{1}, F_{2}\right\}, M\right)$ where $F_{1} \subseteq M \subseteq F_{2}, F_{1}, F_{2} \in \mathcal{F}$ and $|M|=\lceil n / 2\rceil$ (note that $M$ is not necessarily a member of $\mathcal{F}$ ). For every pair $F_{1} \subseteq F_{2}$, there is at least one such $M$ by the assumption on the sizes of elements in $\mathcal{F}_{1}$ and $\mathcal{F}_{2}$, thus there are at least $c\left(P_{2}, \mathcal{F}\right)$ such pairs. On the other hand there are $\left(\begin{array}{c}n \\ \lceil n / 2\rceil\end{array}\right)$ possible $M$ 's. For a fixed $M$ there cannot exist four sets $F_{1}, F_{1}^{\prime}, F_{2}, F_{2}^{\prime} \in \mathcal{F}$ with $F_{1}, F_{1}^{\prime} \subseteq M \subseteq F_{2}, F_{2}^{\prime}$, as they would form a copy of $B$. Thus the number of such $\left(\left\{F_{1}, F_{2}\right\}, M\right)$ for a fixed $M$ is at most the maximum possible size of an antichain in $2^{[\lceil n / 2\rceil]}$. By Theorem 1.1, this is $\left(\begin{array}{c}{[n / 2\rceil} \\ \lceil n / 4\rceil\end{array}\right)$. This finishes the proof of the claim.

To finish the proof of Theorem 5.1, note that by Claim 5.2 it is enough to consider $\left\{P_{3}, B\right\}$-free families $\mathcal{F}$. One can partition the 2-chains contained by such an $\mathcal{F}$ according 
to whether they contain a minimal element $F$ with $|F|>\lfloor n / 2\rfloor$ or a maximal element $F^{\prime}$ with $\left|F^{\prime}\right|<\lceil n / 2\rceil$. The number of such 2-chains is at most $\left(\begin{array}{c}n \\ {[n / 2\rceil}\end{array}\right)\left(\begin{array}{c}{[n / 2\rceil} \\ \lceil n / 4\rceil\end{array}\right)$ by Claim 5.3, while the number of all other 2-chains in $\mathcal{F}$ is at most $\left(\begin{array}{c}n \\ \lceil n / 2\rceil\end{array}\right)\left(\begin{array}{l}{[n / 2\rceil} \\ \lceil n / 4\rceil\end{array}\right)$. This can be seen by applying Claim 5.4 to $\mathcal{F}$ after removing all small maximal and large minimal elements. This finishes the proof of Theorem 5.1.

To obtain Theorem 1.14, one has to compare $\left(\begin{array}{c}n \\ \lceil n / 2\rceil\end{array}\right)\left(\begin{array}{c}\lceil n / 2\rceil \\ \lceil n / 4\rceil\end{array}\right)$ and $\left(\begin{array}{c}n \\ \lfloor n / 3\rfloor\end{array}\right)\left(\begin{array}{c}\lceil 2 n / 3\rceil \\ \lceil n / 3\rceil\end{array}\right)$. The former is clearly not more than $2^{3 n / 2}$ and the latter, by Stirling's formula, is $\Omega\left(\frac{1}{n} 2^{n(2 / 3+h(1 / 3))}\right)$, and $2 / 3+h(1 / 3)=1.58 \ldots$ holds.

\section{Remarks}

One can define an even more general parameter $L a_{R}(P, Q)$. For three posets, $R, P$ and $Q$ we are interested in the maximum number of copies of $Q$ in subposets $R^{\prime}$ of $R$ that do not contain $P$. Analogously to what we had for set families, we say that $R^{\prime} \subseteq R$ is a copy of $Q$ in $R$ if there exists a bijection $\phi: Q \rightarrow R^{\prime}$ such that whenever $x \leq Q x^{\prime}$ holds, then so does $\phi(x) \leq{ }_{R^{\prime}} \phi\left(x^{\prime}\right)$. Let $c(Q, R)$ denote the number of copies of $Q$ in $R$, and for any three posets $R, P$ and $Q$ we define

$$
L a_{R}(P, Q)=\max \left\{c\left(Q, R^{\prime}\right): R^{\prime} \subseteq R, c\left(P, R^{\prime}\right)=0\right\} .
$$

For a poset $R$ and families of posets $\mathcal{P}, \mathcal{Q}$ let us define

$$
L a_{R}(\mathcal{P}, \mathcal{Q})=\max \left\{\sum_{Q \in \mathcal{Q}} c\left(Q, R^{\prime}\right): R^{\prime} \subseteq R, \forall P \in \mathcal{P} \quad c\left(P, R^{\prime}\right)=0\right\} .
$$

Note that $L a(n, P, Q)=L a_{B_{n}}(P, Q)$, where $B_{n}$ is the poset with elements of $2^{[n]}$ ordered by inclusion. Very recently Guo, Chang, Chen, and Li [17] introduced $L a_{R}\left(Q, P_{1}\right)$, as a general approach to forbidden subposet problems. That is, to solve the analogous question in a less complicated structure like the cycle, chain or double chain, and then to apply an averaging argument.

In many parts of Theorem 1.6, the construction yielding the lower bound that matches the upper bound contained the empty set and/or the set $[n]$. One might wonder whether the Lafunction remains the same if we do not allow these elements to be included. In other words, if $B_{n}^{-}$denotes the subposet of $B_{n}$ with $\emptyset$ and $[n]$ removed, then how $L a_{B_{n}^{-}}\left(\vee, P_{2}\right)$ relates to $L a\left(n, \vee, P_{2}\right), L a_{B_{n}^{-}}\left(B, P_{3}\right)$ to $L a\left(n, B, P_{3}\right)$ and so on. The $\{\bigvee, \wedge\}$-free construction $\left(\begin{array}{c}{[n-1]} \\ \lfloor(n-1) / 2\rfloor\end{array}\right) \cup\left\{F \cup\{n\}: F \in\left(\begin{array}{c}{[n-1]} \\ \lfloor(n-1) / 2\rfloor\end{array}\right)\right\}$ and the $B$-free construction $\left(\begin{array}{c}{[n-2]} \\ \lfloor(n-2) / 2\rfloor\end{array}\right) \cup\{F \cup\{n-1\}$ : $\left.F \in\left(\begin{array}{c}{[n-2]} \\ \lfloor(n-2) / 2\rfloor\end{array}\right)\right\} \cup\left\{F \cup\{n-1, n\}: F \in\left(\begin{array}{c}{[n-2]} \\ \lfloor(n-2) / 2\rfloor\end{array}\right)\right\}$ show that

- $\quad\left(\begin{array}{c}n-1 \\ \lfloor(n-1) / 2\rfloor\end{array}\right) \leq L a_{B_{n}^{-}}\left(\bigvee, P_{2}\right)=L a_{B_{n}^{-}}\left(\bigwedge, P_{2}\right) \leq\left(\begin{array}{c}n \\ \lfloor n / 2\rfloor\end{array}\right)$,

- $\quad\left(\begin{array}{c}n-2 \\ \lfloor(n-2) / 2\rfloor\end{array}\right) \leq L a_{B_{n}^{-}}\left(B, P_{3}\right) \leq\left(\begin{array}{c}n \\ \lfloor n / 2\rfloor\end{array}\right)$.

There is a longstanding (folklore) conjecture which would imply the existence of constructions in both cases that asymptotically match the upper bounds. Let $\mathcal{M}_{k+1} \subseteq\left(\begin{array}{c}{[n]} \\ k+1\end{array}\right)$ be a family of sets with the property that for every $K \in\left(\begin{array}{c}{[n]} \\ k\end{array}\right)$ there exists at most one set $M \in \mathcal{M}_{k+1}$ with $K \subsetneq M$. Obviously, for any such set we have $\left|\mathcal{M}_{k+1}\right| \leq\left(\begin{array}{l}n \\ k\end{array}\right) /(k+1)$ and $\mathcal{R}_{k+1}:=\mathcal{M}_{k+1} \cup\left(\begin{array}{c}{[n]} \\ k\end{array}\right)$ is $\bigvee$-free with $c\left(P_{2}, \mathcal{R}_{k+1}\right)=(k+1)\left|M_{k+1}\right|$. It is conjectured that there exists a family $\mathcal{M}_{\lfloor n / 2\rfloor+1}$ with the above property such that $\left|\mathcal{M}_{\lfloor n / 2\rfloor+1}\right|=(1-$ 
$o(1))\left(\begin{array}{c}{[n]} \\ \lfloor n / 2\rfloor\end{array}\right) /(\lfloor n / 2\rfloor+1)$ holds. Similarly, writing $\overline{\mathcal{M}}_{n-k+1}$ for $\left\{[n] \backslash M: M \in \mathcal{M}_{n-k+1}\right\}$, the construction $\mathcal{T}_{k}:=\mathcal{M}_{k+1} \cup\left(\begin{array}{c}{[n]} \\ k\end{array}\right) \cup \overline{\mathcal{M}}_{n-k+1}$ is $B$-free. The above conjecture would yield $c\left(P_{3}, \mathcal{T}_{\lfloor n / 2\rfloor}\right)=(1-o(1))\left(\begin{array}{c}n \\ \lfloor n / 2\rfloor\end{array}\right)$.

In Section 4, we proved that, apart from a polynomial factor, Conjecture 1.5 holds for chains of length $l+1$ and complete $l$-level posets, i.e. there exists a sequence $\mathcal{F}_{n}$ of families that consists of full levels such that $L a\left(n, P_{l+1}, K_{r_{1}, r_{2}, \ldots, r_{l}}\right) \leq n^{k} c\left(\mathcal{F}_{n}, K_{r_{1}, r_{2}, \ldots, r_{l}}\right)$ for some constant $k=k\left(K_{r_{1}, r_{2}, \ldots, r_{l}}\right)$. To improve this result or to completely get rid of the polynomial factor one would need to improve Theorem 1.8 or rather to determine the intersection profile polytope of antichains.

Funding Information Open access funding provided by MTA Alfréd Rényi Institute of Mathematics (MTA RAMKI)

Open Access This article is distributed under the terms of the Creative Commons Attribution 4.0 International License (http://creativecommons.org/licenses/by/4.0/), which permits unrestricted use, distribution, and reproduction in any medium, provided you give appropriate credit to the original author(s) and the source, provide a link to the Creative Commons license, and indicate if changes were made.

\section{References}

1. Alon, N., Shikhelman, N.: Many $T$-copies in $H$-free graphs. J. Combinatorial Theory, Ser. B 121, 146172 (2016)

2. Bollobás, B.: Sperner systems consisting of pairs of complementary subsets. Journal of Combinatorial Theory, Series A 15, 363-366 (1973)

3. Bukh, B.: Set families with a forbidden subposet. Electronic Journal of Combinatorics 16(1), P142 (2009)

4. Burcsi, P., Nagy, D.: The method of double chains for largest families with excluded subposets. Electronic Journal of Graph Theory and Applications 1, 40-49 (2013)

5. DeBonis, A., Katona, G.O.H., Swanepoel, K.: Largest family without $A \cup B \subset C \cap D$. J. Combin. Theory Ser. A 111, 331-336 (2005)

6. Erdös, P.: On a lemma of Littlewood and Offord. Bull. Am. Math. Soc. 51, 898-902 (1945)

7. Engel, K.: Sperner Theory. Cambridge University Press (1997)

8. Erdös, P.L., Frankl, P., Katona, G.O.H.: Intersecting Sperner families and their convex hulls. Combinatorica 4, 21-34 (1984)

9. Erdös, P.L., Frank1, P., Katona, G.O.H.: Extremal hypergraph problems and convex hulls. Combinatorica 5, 11-26 (1985)

10. Erdös, P.L., Frankl, P., Katona, G.O.H.: Convex hulls of more-part Sperner families. Graphs and Combinatorics 2, 123-134 (1986)

11. Frankl, P., Katona, G.O.H.: Polytopes determined by hypergraph classes. European J. Combin. 6, 233243 (1985)

12. Gerbner, D., Methuku, A., Nagy, D.T., Patkós, B., Vizer, M.: On the number of containments in P-free families. arXiv: 1804.01606

13. Gerbner, D., Patkós, B.: L-chain profile vectors. SIAM J. Discrete Math. 22, 185-193 (2008)

14. Gerbner, D., Patkós, B.: Extremal Finite Set Theory. 1st Edition CRC Press (2018)

15. Griggs, J.R., Li, W.-T.: Progress on poset-free families of subsets. In: Recent Trends in Combinatorics, pp. 317-338 (2016)

16. Griggs, J.R., Lu, L.: On families of subsets with a forbidden subposet. J. Combinatorial Theory (Ser. A) 119, 310-322 (2012)

17. Guo, J.-Y., Chang, F.-H., Chen, H.-B., Li, W.-T.: Families of Subsets Without a Given Poset in the Interval Chains. arXiv:1605.00373

18. Katona, G.: A Theorem of Finite Sets. Theory of Graphs, Proc. Coll. Held at Tihany, Hungary (1966)

19. Katona, G.O.H.: Two application of Sperner type theorems (For search theory and truth functions). Period. Math. Hungar. 3, 19-26 (1973) 
20. Katona, G.O.H.: Convex Hulls of Hypergraph Classes, Proc. of the Fourth Yugoslav Seminar on Graph Theory, Novi Sad, 1983, pp. 177-190. Univ. Novi Sad, Novi Sad (1984)

21. Katona, G.O.H., Tarján, T.: Extremal problems with excluded subgraphs in the $n$-cube, Graph Theory, Lagow Lecture Notes in Math. 1018, pp. 84-93. Springer, Berlin (1983)

22. Kruskal, J.B.: The number of simplices in a complex. Math. Optimization Tech, pp. 251-278 (1963)

23. Methuku, A., Tompkins, C.: Exact forbidden subposet results using chain decompositions of the cycle. Electron. J. Combin. 22, 4.29 (2015)

24. Patkós, B.: The distance of $\mathcal{F}$-free families. Stud. Sci. Math. Hung. 46(2), 275-286 (2009)

25. Sperner, E.: Ein Satz über Untermengen einer endlichen Menge. Math. Z. 27, 544-548 (1928)

Publisher's Note Springer Nature remains neutral with regard to jurisdictional claims in published maps and institutional affiliations. 